\title{
The leading-edge vortex of yacht sails
}

\author{
Abel Arredondo-Galeana, Ignazio Maria Viola* \\ School of Engineering, Institute for Energy Systems, University of Edinburgh, Mayfield Road, Edinburgh, EH9 3DW, UK
}

\begin{abstract}
It has been suggested that a stable Leading Edge Vortex (LEV) can be formed from the sharp leading edge of asymmetric spinnakers, which are high-lift sails used by yachts to sail downwind. If the LEV remains stably attached to the leading edge, it provides an increase in the thrust force. Until now, however, the existence of a stable and attached LEV has only been shown by numerical simulations. In the present work we experimentally verify, for the first time, that a stable LEV can be formed on an asymmetric spinnaker. We tested a 3D printed rigid sail in a water flume at a chord-based Reynolds number of ca. $10^{4}$. The sail was tested in isolation without hull and rigging. The flow field was measured with Particle Image Velocimetry (PIV) over horizontal cross sections. We found that on the leeward side of the sail (the suction side), the flow separates at the leading edge reattaching further downstream and forming a stable LEV. The LEV grows in diameter from the root to the tip of the sail, where it merges with the tip vortex. We detected the LEV using the $\gamma$ criterion, and we verified its stability over time. The lift contribution provided by the LEV was computed solving a complex potential model of each sail section. This analysis indicated that the LEV provides more than $10 \%$ of the total sail's lift. These findings suggest that the maximum lift of lowaspect-ratio wings with a sharp leading edge, such as spinnakers, can be enhanced by promoting the formation of a stable LEV.
\end{abstract}

Keywords: Yacht sails, Spinnakers, Vortex Flows, Leading Edge Vortex, Particle Image Velocimetry, Circular arc

\section{Introduction}

Sails are thin wings with a relatively sharp leading edge. A common configuration for downwind sailing includes two sails: the mainsail and the spinnaker (Fig. 1). The mainsail, which is on the rear of the yacht, has both the leading edge and the lower edge attached to rigid structures (the mast and the boom, respectively). Conversely, the spinnaker, which is in the front of the yacht, is attached to the boat only by the three corners. The free, sharp leading edge leads to flow separation at any non-zero angle of attack (Fig. 2). This is one of the key features of yacht sails that makes them different from conventional wings. In fact, the flow at the leading edge is similar to that of a plate at incidence (Viola and Flay, 2015). Flow reattachment occurs somewhere downstream of the leading edge, forming a region of separated flow. This region is short in the chordwise direction, but it extends from the base to the tip of the sail (Viola et al., 2013). When sailing

\footnotetext{
*Corresponding author. Tel: +44 1316505622

Email address: I.M.Viola@ed.ac.uk (Ignazio Maria Viola)
}

downwind, the most efficient fore sails are asymmetric spinnakers, which are highly-cambered, highly-twisted and low-aspect-ratio sails. The maximum camber in both the chordwise and spanwise directions is typically higher than $20 \%$ and $50 \%$ of the chord length, respectively. The twist angle between the root and top section is higher than $20^{\circ}$, and the aspect ratio is between 1.5 and 2 . These sails are designed to allow the maximum lift, and the drag has little effect on the yacht performance because it is almost perpendicular to the sail course.

The large camber enables high lift, but it also leads to trailing edge separation. The rear separated region could cover more than half of the chord. Since the extent of it is easier to identify than the smaller leading edge separated area, the length of the rear region is typically used to inform the sail designer on where the sails' shape can be enhanced. However, virtually all of the driving force is generated near the leading edge. Thus, small changes in the fluid dynamics of the leading edge separated region can result in significant gains in performance. This work aims to gain new insight on the flow in this section. 
The impact of this work, however, extends beyond sail design. In particular, there is an increasing interest for very thin wings, and also membrane-wings, for Unmanned Aerial Vehicles (UAV). These operate at moderate Reynolds numbers $(R e)$ of the order of $10^{4}-10^{5}$. At this $R e$ regime, either a large angle of attack or a high camber must be used to generate lift. Therefore, the flow around this highly cambered sail is relevant for the design of UAV wings.

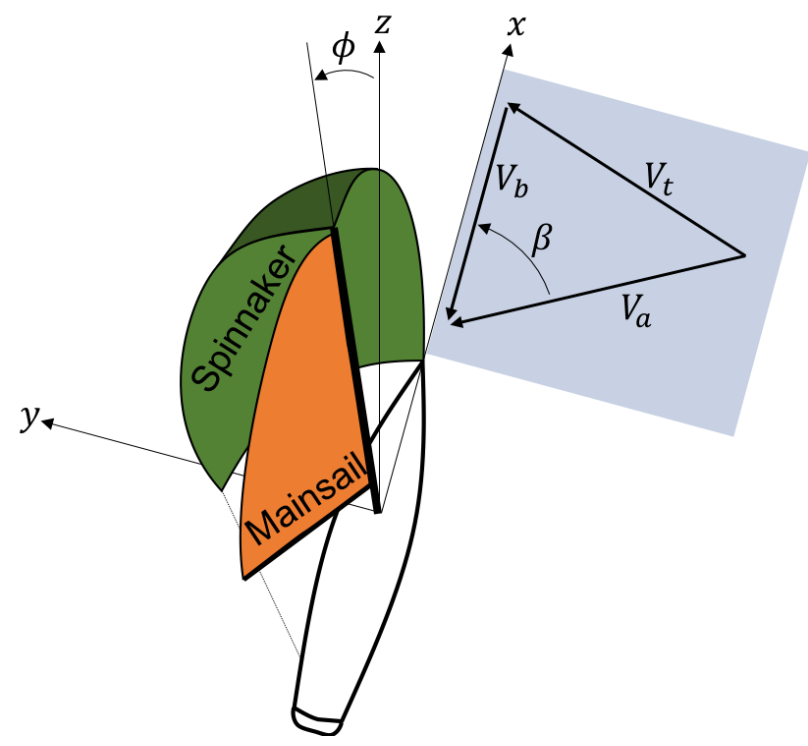

Figure 1: Bird eye view of a yacht sailing downwind, where $\phi$ is the heel angle in the vertical plane perpendicular to the yacht, $V_{b}$ is the wind due to the boat speed, while $V_{t}$ and $V_{a}$ are the true and apparent wind, respectively; $\beta$ is the apparent wind angle

\subsection{The flow of sharp-edge sails}

When the leading edge of a wing is sharp and the incidence angle is high, the flow separates forming a strong shear layer. This results in the production of vorticity that is accumulated in the separated region. The integral of the vorticity in this region leads to a circulation that has the same sign as the circulation of the sail; thus this vorticity contributes to the generation of lift. However, vorticity cannot be accumulated indefinitely. It can be either shed downstream with the main flow stream, or it must be somehow extracted. At the leading edge of genoas and jibs, which are higher aspect ratio sails than spinnakers and are used to sail upwind, the vorticity is continuously shed downstream in the form of vortices that roll on the surface of the airfoil toward the trailing edge (Viola and Flay, 2011a, 2015; Nava et al., 2016). The time-averaged flow field shows flow reattachment somewhere downstream of the leading edge, and a thick boundary layer that grows towards the trailing edge.

Recent Detached Eddy Simulations (DES) (Viola et al., 2014) have revealed that a stable attached LEV might also occur on the asymmetric spinnakers of sailing yachts. This was anecdotally anticipated by Bethwaite (Bethwaite, 1993), who sketched the LEV on the asymmetric spinnaker of high-performance dinghies. The LEV is a coherent vortex formed by the roll up of vorticity, generated at the leading edge. The vorticity is not continuously shed downstream, but is instead convected towards the centre of the vortex. If the vorticity is somehow extracted from the axis of the vortex, it is possible to achieve a stable LEV that remains attached to the leading edge indefinitely. The vorticity is typically extracted by axial flow inside of the vortex core, towards the wing tip. A stable LEV grows in the direction in which the vorticity is extracted. The vorticity and circulation of the LEV can significantly increase the lift and thus it is exploited on both man-made and natural flyers (Ellington, 1999; Srygley and Thomas, 2002; Garmann et al., 2013; Jardin and David, 2014). Remarkably, it has been identified across a wide range of $R e$. In laminar flow conditions, it has been found on autorotating seeds (Lentink et al., 2009), and on the wings of insects (Muijres et al., 2008) and small birds (Lentink et al., 2007). In transitional and turbulent flow conditions, it has been found on larger bird wings (Hubel and Tropea, 2010), fish fins (Borazjani and Daghooghi, 2013) and delta wings (Gursul et al., 2005, 2007). In helicopter rotors (Corke and Thomas, 2015) and wind turbines (Larsen et al., 2007), the LEV is a powerful but undesirable flow feature. This is due to the large angle of attack oscillations. At every period, the LEV is shed downstream leading to a lift overshoot above the quasi-static maximum lift and to an abrupt, and dangerous change in the pitching moment. Conversely, in biological flyers and delta wings, the LEV provides an essential source of lift augmentation.

This work aims to provide experimental evidence that a stable LEV can occur on asymmetric spinnakers, corroborating the numerical evidence. Moreover, the work aims to quantify the contribution of the LEV to sails' performances.

\subsection{A Benchmark for Downwind Sails}

The asymmetric spinnaker where the LEV was identified with DES (Viola et al., 2014) is considered in this work. The aerodynamics of this sail have been 


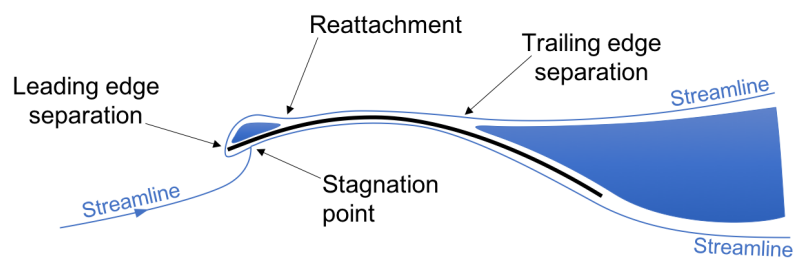

Figure 2: Schematic view of the flow over a horizontal section of a spinnaker

widely investigated in the last decade and this makes it one of the best available benchmarks for downwind sails. The geometry and the experimental, and numerical data are available on the Edinburgh DataShare (datashare.is.ed.ac.uk). This sail was designed for the AC33 class, which was proposed for the $33^{\text {rd }}$ America's Cup. This class has never been adopted, as the $33^{\text {rd }}$ America's Cup was eventually disputed with multi-hulls (ruled by the Deed of Gift). A $1: 15^{\text {th }}$-scale model of this sail was tested in a wind tunnel at $55^{\circ}$ apparent wind angle and $10^{\circ}$ heel angle. The apparent wind angle is the supplementary angle between the wind velocity experienced by the yacht and the sailed course. The forces (Viola and Flay, 2009) and pressures (Viola and Flay, 2010) on the sail surfaces were recorded for a range of sail trims, and also compared with those measured on similar sails. The sail trim that allowed the maximum driving force, was used to build a rigid sail with embedded pressure taps and both forces, and pressures were measured in a wind tunnel (Bot et al., 2014). This sail trim was also modelled with Reynolds-averaged NavierStokes (RANS) simulations (Viola and Flay, 2011b) and with DES (Viola et al., 2014). A $1: 3^{\text {rd }}$-scale prototype was built and tested on water on a Platu25-class yacht (Viola and Flay, 2012), where surface pressures were measured. A three-way comparison between the pressures measured in a wind tunnel, on water and with RANS was presented in Viola and Flay (2011b). While a comparison between wind tunnel tests performed with flexible and rigid sails, and DES, was presented in both Bot et al. (2014) and Viola et al. (2014). The pressures from these three approaches showed a qualitative agreement, with the pressures computed numerically lying in between those measured with the two experimental techniques.

\subsection{Overview of the Present Work}

In order to test in highly controlled flow conditions and to identify the main mechanisms enabling the for- mation, and stability of the LEV, the asymmetric spinnaker is tested in isolation (without the mainsail and the hull). Consider the chord measured on a section at $3 / 4^{\text {th }}$ of the mitre from the base of the sail, where the mitre is the line on the sail surface equally far from the leading and trailing edge. Based on this reference chord, the Reynolds number of the sail tested in this work is $R e=1.3 \times 10^{4}$. The actual flow of a real sail is certainly more complex than the one of this simplified model. The enhanced turbulent mixing and boundary layer effects at higher Reynolds numbers are not accounted for in this model. However, the LEV has been found to be very resilient to the effects of Reynolds numbers (Gordnier et al., 2009). Also, the effect of the other sails and the hull is neglected. The rear sail generates upwash and increases the effective angle of attack. Therefore, these effects can be mostly accounted for by adjusting the angle of attack. The proximity to the hull and the sea surface leads to a local variation of the flow near the bottom of the sail. The effect of this variation is marginal on the LEV because the LEV is mostly developed at the highest sections of the sail. The onset flow is uniform and does not take into account the variation in height of the apparent wind velocity experienced by a sail at full scale. However, also this effect is moderate and can mostly be accounted for by a different trim of the sail. For this reason, the benchmark experiments and simulations described in section 1.2 consider a uniform onset flow. Therefore, while this investigation does not provide a quantitative description of the full-scale flow, it enables the understanding of the key features of downwind sail flow.

The rest of the paper is organised as follows: in Section 2, Methodology, the details of the methodology is presented, including the geometry of the sail, the experimental rig, the flow conditions, the instrumentation used to measure the flow field and how the data is analysed. In Section 3, Results, the flow measurements are presented, including the analysis of the LEV and an estimate of the contribution of the LEV to the sail's performance. Finally, Section 4, Conclusions, the key findings are summarised.

\section{Methodology}

\subsection{Sail Model}

The geometry of the 3D-printed model used for this investigation is available on www.ignazioviola.com. The model has an area of $A=0.045 \mathrm{~m}^{2}$. The twist angle from the base to the head is $16^{\circ}$, the maximum chordwise camber is $0.40 c_{0}$ and the maximum spanwise cam- 


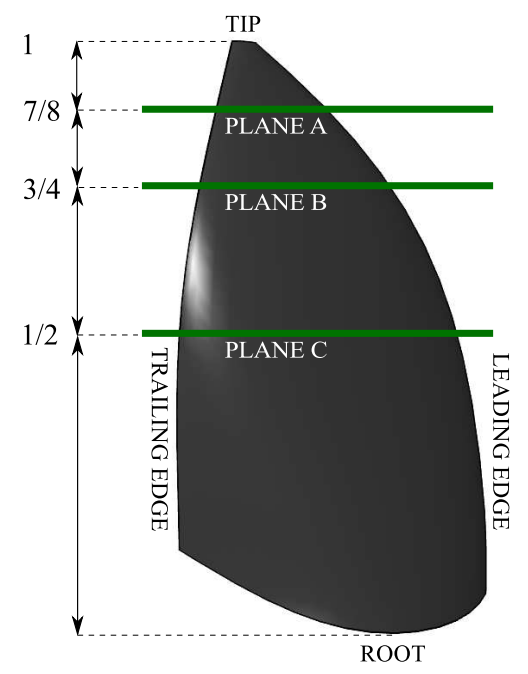

Figure 3: Rendering of the sail model and position of the measurement planes

ber is $0.65 c_{0}$, where $c_{0}=0.114 \mathrm{~m}$ is the chord of a sail section at $3 / 4^{\text {th }}$ of the span from the base (Fig. 3).

The model is $3 \mathrm{~mm}$ thick. Separation at the leading edge is promoted chamfering the edges. The chamfer at the leading and trailing edges is $20^{\circ}$ for the first $3 / 4^{\text {th }}$ of the span from the base to the head. At the top $1 / 4^{\text {th }}$ of the sail's span, the chamfer grows progressively from $20^{\circ}$ to $70^{\circ}$, allowing the thickness to remain constant on the mitre. The head of the sail is blunt.

The model is 3D printed in ABS with a Fortus 250 3D printer. It is mounted on a rotating shaft controlled by a lever arm for the fine control of the angle of attack. The shaft is attached to a $6 \mathrm{~mm}$ thick acrylic plate connected to a pair of $45 \times 45 \mathrm{~mm}$ aluminium extrusions attached to the flume's side walls. The rig allows to change the angle of attack and to secure its testing position through an arch dial system (Fig. 4). The shaft is set to replicate the same apparent wind angle $\left(55^{\circ}\right)$ and heel angle $\left(10^{\circ}\right)$ as tested with DES (Viola et al., 2014).

\subsection{Water Flume}

The water flume is a current-wave testing facility in the Institute of Engineering Systems of the School of Engineering, University of Edinburgh. It is $2 \mathrm{~m}$ long, $0.4 \mathrm{~m}$ wide and $0.9 \mathrm{~m}$ high. The water depth is set to $0.5 \mathrm{~m}$ and the sail is placed horizontally $0.1 \mathrm{~m}$ below the water surface. The free space between the rig and the walls of the flume is $0.05 \mathrm{~m}$ at both sides. The model is tested in a uniform current with $U_{\infty}=0.1146 \mathrm{~m} / \mathrm{s}$. A turbulence intensity of $7 \%$ is measured with laser
Doppler velocimetry at a location $1 \mathrm{~m}$ upstream of the model. The high level of turbulence is due to the lack of a contraction section, and it is similar to that experienced by a yacht at full scale. Therefore, the vortical flow structures here investigated convect within a turbulent stream as they would do at full scale. As shown by McWilliams (1984), coherent vortices are highly resilient to the turbulent perturbations.

\subsection{Particle Image Velocimetry}

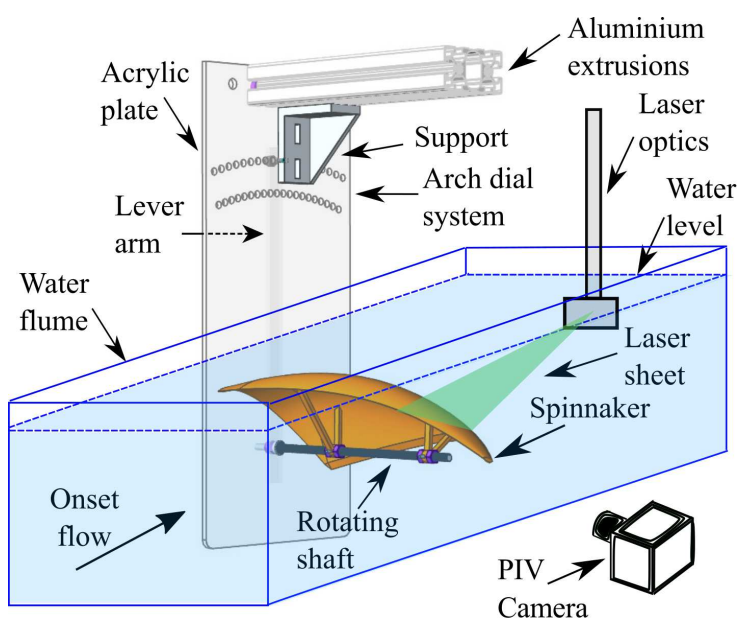

Figure 4: Schematic diagram of the experimental setup

Flow visualisation is performed with a Particle Image Velocimetry (PIV) system, which consists of a Solo 200XT pulsed dual-head Nd:YAG laser, with an energy output of $200 \mathrm{~mJ}$ at a wavelength of $\lambda=532 \mathrm{~nm}$. The camera is a CCD Imperx 5MP with a 2448 px $\times 2050$ px resolution and a Nikkor $\mathrm{f} / 2,50 \mathrm{~mm}$ lense. The seeding particles are silver coated hollow glass spheres with an average diameter of $14 \mu \mathrm{m}$ and a density of $1.7 \mathrm{~g} / \mathrm{cc}$. In order to mitigate surface reflections, a coating of matt black paint is applied to the sail with a second coating of rhodamine B. A third coating of acrylic is applied to protect the rhodamine B coating from the water. An optical filter is used on the camera to subtract the wavelength of rhodamine B and minimise the reflected light. Background subtraction (Wereley et al., 2002) enables measurements in close proximity to the wall. The leading edge region, however, is not affected by laser reflections due to the curvature of the sail and the direction of the laser sheet.

The laser beam is redirected through two mirrors and an array of underwater LaVision optics to generate a laser sheet parallel to the flow. The laser sheet is fully submerged as shown in Fig. 4. The thickness of the 
laser sheet is approximately $2 \mathrm{~mm}$. Three cross sections of the sail are recorded: plane A, B and C (Fig. 3). These are located respectively at $7 / 8,3 / 4$ and $1 / 2$ of the distance from the root of the sail to the tip.

PIV pair images are sampled at $7.5 \mathrm{~Hz}$. A two pass adaptive correlation is applied. The first pass has a $64 \mathrm{px} \times 64 \mathrm{px}$ interrogation window, with a Gaussian weighting and $50 \%$ window overlap. The second pass has a $24 \mathrm{px} \times 24 \mathrm{px}$ interrogation window and a $75 \%$ window overlap. Averaged fields are generated from the full time series and a $3 \times 3$ filter is used to smoothen the vector fields.

\subsection{Vortex Detection Criteria}

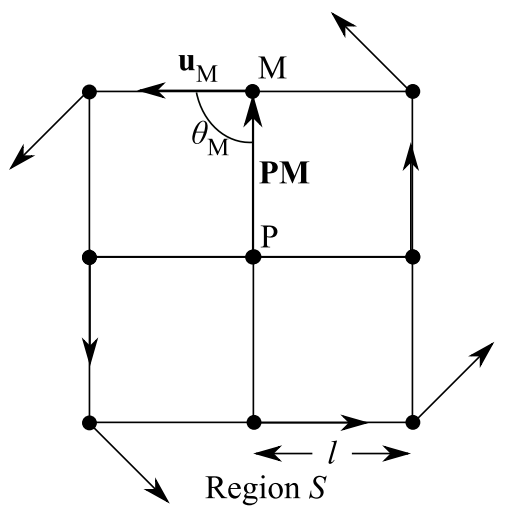

Figure 5: Vortex detection algorithm

Vortices are detected with the $\gamma_{1}$ and the $\gamma_{2}$ criteria (Graftieaux et al., 2001), which have been successfully applied to PIV data (Rabinovitch et al., 2012; Harbig et al., 2013; Pitt Ford and Babinsky, 2013). The $\gamma_{2}$ criterion is the non-Galilean invariant version of the $\gamma_{1}$ criterion, as the local convection velocity is subtracted. Figure 5 shows a schematic drawing of the $\gamma_{1}$ algorithm. The $\gamma_{1}$ criterion at a point $\mathrm{P}$ is computed using the PIV data within a square window $S$ of size $2 l \times 2 l$ centred in P. At each point $\mathrm{M}$ within $S$, the sine of the angle $\theta_{\mathbf{M}}$ between the vector $\mathbf{P M}$ and the velocity $\mathbf{u}_{\mathbf{M}}$ is computed. The $\gamma_{1}$ value in $P$ is given by

$$
\gamma_{1}=\frac{1}{N} \sum_{S} \frac{\mathbf{P M} \times \mathbf{u}_{\mathbf{M}}}{\|\mathbf{P M}\| \cdot\left\|\mathbf{u}_{\mathbf{M}}\right\|}=\frac{1}{N} \sum_{S} \sin \left(\theta_{\mathbf{M}}\right),
$$

where $N$ is the number of grid points in $S$.

In the $\gamma_{2}$ criterion, the average convection velocity $\langle\mathbf{u}\rangle$ in the region $S$ is subtracted from every velocity point in the interrogation window, such that

$$
\gamma_{2}=\frac{1}{N} \sum_{S} \frac{\mathbf{P M} \times\left(\mathbf{u}_{\mathrm{M}}-\langle\mathbf{u}\rangle\right)}{\|\mathbf{P M}\| \cdot\left\|\mathbf{u}_{\mathrm{M}}-\langle\mathbf{u}\rangle\right\|},
$$

where

$$
\langle\mathbf{u}\rangle=\frac{1}{N} \sum_{S} \mathbf{u}_{\mathrm{M}}
$$

\subsection{Calibration of the Vortex Detection Criteria}

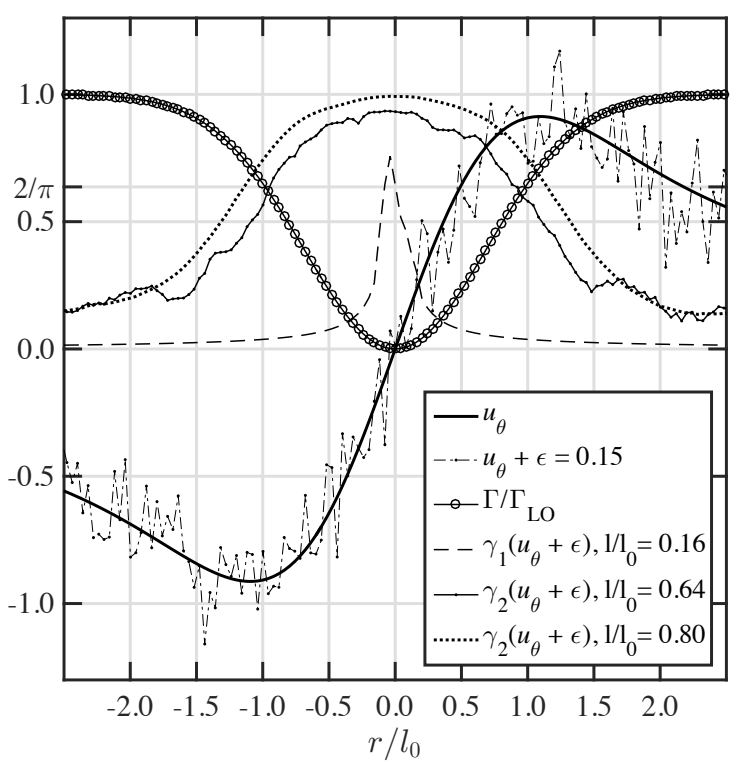

Figure 6: $\gamma_{1}$ and $\gamma_{2}$ criteria for a Lamb-Oseen vortex

To calibrate the $\gamma_{1}$ and $\gamma_{2}$ criteria, these are tested on an isolated Lamb-Oseen vortex. The tangential velocity of the Lamb-Oseen vortex is

$$
u_{\theta}=\frac{\Gamma_{\mathrm{LO}}}{2 \pi r}\left(1-\exp \left(-\frac{r^{2}}{l_{0}^{2}}\right)\right),
$$

where $\Gamma_{\mathrm{LO}}$ is the strength of the vortex, $r$ is the radial coordinate and $l_{0}$ is the core vortex radius, defined as the radial coordinate where the tangential velocity is maximum. Its circulation is given by

$$
\Gamma=\Gamma_{\mathrm{LO}}\left(1-\exp \left(-\frac{r^{2}}{l_{0}^{2}}\right)\right) .
$$

The $\gamma_{1}$ and $\gamma_{2}$ criteria for the Lamb-Oseen vortex are shown in Fig. 6. The $\gamma_{2}$ criterion is computed for two different sizes $l$ of the interrogation window $S$ : $l / l_{0}=0.64$ and 0.80 ; for $\gamma_{1}, l / l_{0}=0.16$. The centre of the vortex is identified by the maximum of both the $\gamma_{1}$ and $\gamma_{2}$ criteria, while the radius of the vortex core is identified by $\left|\gamma_{2}\right|=2 / \pi$.

A random error $\epsilon=15 \% u_{\theta}$ is included to model the effect of PIV noise that is generated during acquisition and post-processing (Morgan et al., 2009). A 
$15 \%$ noise in $\gamma_{1}$ is found to decrease the magnitude of the detection peak by $30 \%$. When $l / l_{0}$ decreases, $\gamma_{2}$ shows greater fluctuations and it behaves more like a local criterion. This leads to the underestimation of the vortex core size. For the $\gamma_{1}$ criterion, the smaller the $l / l_{0}$ is set, the narrower the detection peak becomes (Graftieaux et al., 2001). Varying the window size of the $\gamma_{2}$ criterion results in different $\gamma_{2}$ contours, but the threshold $\left|\gamma_{2}\right|=2 / \pi$ that corresponds to the core size of the Lamb-Oseen vortex is almost independent of the window size.

In the present experiment, the LEV core size is found to be ca. $l_{0}=0.1 c$. The size $l$ of the interrogation window is set to $l / l_{0}=0.16$ and 0.80 for $\gamma_{1}$ and $\gamma_{2}$, respectively. The noise level in the experiment is estimated to be ca. $15 \% u_{\theta}$. In fact, the maximum $\left|\gamma_{1}\right|$ on the sail is 0.7 .

\subsection{Complex Potential Model}

In order to estimate the contribution of the LEV to the lift of the sail, a potential flow model of a circular arc is developed. The arc has the same chord $c$ and maximum camber $2 \mu$ than the considered sail's section, and it experiences a uniform flow with the same free stream velocity $U_{\infty}$ and angle of attack $\alpha$ with respect to the chord. The arc can be mapped onto a rotating circular cylinder whose external flow has the same circulation than the arc. The cylinder is defined in the complex plane $\zeta$, where the complex coordinate

$$
\zeta \equiv X+i Y \equiv r e^{i \theta}
$$

identifies a position vector in the Cartesian coordinates $(X, Y)$ and in the polar coordinates $(r, \theta)$. The velocity potential $\phi=\phi(\zeta)$ and the stream function $\psi=\psi(\zeta)$ are such that the velocity in the $X$-direction is

$$
U \equiv \frac{\partial \phi}{\partial X} \equiv \frac{\partial \psi}{\partial Y}
$$

and the velocity in the $Y$-direction is

$$
V \equiv \frac{\partial \phi}{\partial Y} \equiv-\frac{\partial \psi}{\partial X}
$$

The complex potential is

$$
F(\zeta) \equiv \phi(\zeta)+i \psi(\zeta)
$$

and the complex velocity is

$$
W(\zeta) \equiv \frac{\partial F(\zeta)}{\partial \zeta}=U-i V .
$$

The complex velocity provides the velocity field around the rotating cylinder. The cylinder is centred in $\zeta_{0}=\mu e^{i \pi / 2}=i \mu$ and has a radius $R=c /(4 \cos \beta)$, where $\beta=\arctan (4 \mu / c)$ (Fig. 7). And the maximum camber is $2 \mu$.

The velocity field in the $\zeta$ plane of the cylinder can be mapped onto the plane $\hat{z}$ of the circular arc with the Joukowski transformation

$$
\hat{z}=\zeta+\frac{(R \cos \beta)^{2}}{\zeta}
$$

In the $\hat{z}$ plane, the coordinate system is centred in the middle of the chord, such that the $\hat{x}$-axis is in the direction of the chord and positive toward the trailing edge, and the $\hat{y}$-axis is positive toward the suction side.

Finally, a further transformation

$$
z=\hat{z} e^{-i \alpha}
$$

allows a description of the flow field in the flume reference system, where the $x$-axis is aligned with the free stream velocity $U_{\infty}$. These two transformations are shown in Fig. 8. is

The complex potential of the cylinder in the $\zeta$ plane

$$
F_{0}(\zeta)=U_{\infty}\left(\zeta-\zeta_{0}\right) e^{-i \alpha}+\frac{U_{\infty} R^{2} e^{i \alpha}}{\zeta-\zeta_{0}}-\frac{i \Gamma_{0}}{2 \pi} \ln \left(\zeta-\zeta_{0}\right),
$$

where

$$
\Gamma_{0}=-4 \pi U_{\infty} R \sin (\alpha+\beta)
$$

is the circulation of the cylinder. Circulation values are defined positive anticlockwise.

The LEV can be modelled as a free vortex in the $\zeta$ plane. The circulation of the free vortex $\Gamma_{\mathrm{LEV}}$ is computed from the measured flow field, as the integral of the tangential velocity over the closed iso-line $\mathbf{I}$ of the $\gamma_{2}$ criterion, where $\gamma_{2}=0.67$ :

$$
\Gamma_{\mathrm{LEV}}=\oint_{\left|\gamma_{2}\right|=0.67} \mathbf{u} \cdot \mathrm{d} \mathbf{l}
$$

A correction is then applied as described in the following Sec. 2.7.

The coordinates of the centre of the LEV, determined with the $\gamma_{2}$ criteria, are used to compute its polar coordinates in terms of $\rho$ and $\tau$ in the $\zeta$ plane

$$
\zeta_{L E V}=\rho e^{i \tau}+\mu e^{i \pi / 2}
$$

If only one free vortex was added, the cylinder would no longer be impermeable and in the $\hat{z}$ plane, the Kutta condition would not be satisfied at the trailing edge. In order to restore the impermeability of the cylinder, a 
mirror vortex with circulation $-\Gamma_{\mathrm{LEV}}$ must be placed inside of the cylinder at the inverse square point

$$
\zeta_{L E V}^{\prime}=\frac{R^{2}}{\rho} e^{i \tau}+\mu e^{i \pi / 2} .
$$

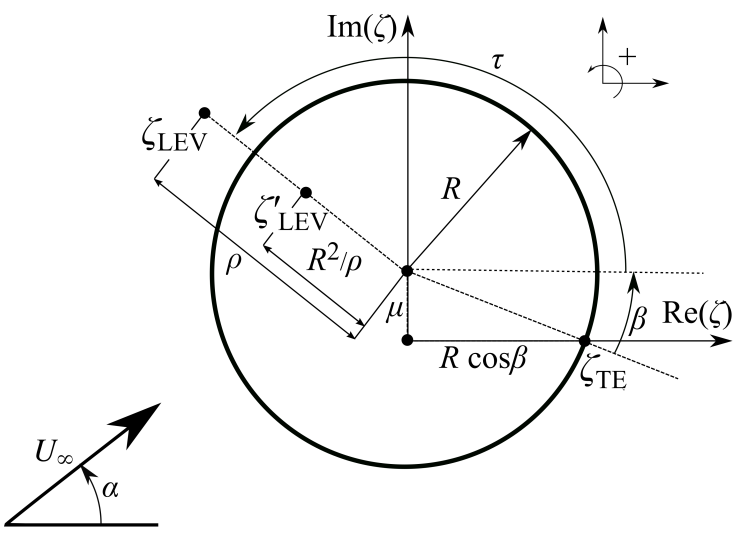

Figure 7: Complex potential model in the $\zeta$ plane

The resulting complex potential is

$$
\begin{aligned}
F(\zeta) & =U_{\infty}\left(\zeta-\zeta_{0}\right) e^{-i \alpha} \\
& +\frac{U_{\infty} R^{2} e^{i \alpha}}{\left(\zeta-\zeta_{0}\right)}-\frac{i\left(\Gamma_{\mathrm{b}}+\Gamma_{\mathrm{LEV}}\right)}{2 \pi} \ln \left(\zeta-\zeta_{0}\right) \\
& -\frac{i \Gamma_{\mathrm{LEV}}}{2 \pi} \ln \frac{\zeta-\zeta_{\mathrm{LEV}}}{\zeta-\zeta_{\mathrm{LEV}}^{\prime}} .
\end{aligned}
$$

where the first line is the contribution of the free stream, the second line is due to the cylinder and the circulation in the centre of the cylinder and the third line is due to the free vortices in $\zeta_{\mathrm{LEV}}$ and $\zeta_{\mathrm{LEV}}^{\prime}$. By derivation of the complex potential, we compute the complex velocity as

$$
\begin{aligned}
W(\zeta) & =U_{\infty} e^{-i \alpha} \\
& -\frac{U_{\infty} R^{2} e^{i \alpha}}{\left(\zeta-\zeta_{0}\right)^{2}}-\frac{i\left(\Gamma_{\mathrm{b}}+\Gamma_{\mathrm{LEV}}\right)}{2 \pi} \frac{1}{\zeta-\zeta_{0}} \\
& -\frac{i \Gamma_{\mathrm{LEV}}}{2 \pi} \frac{\zeta-\zeta_{\mathrm{LEV}}^{\prime}}{\zeta-\zeta_{\mathrm{LEV}}} .
\end{aligned}
$$

Having derived the complex velocity for a generic $\Gamma_{b}$, it is now possible to compute the $\Gamma_{\mathrm{b}}$ that satisfies the Kutta condition. The $\zeta_{T E}$ coordinate, corresponding to the trailing edge of the circular arc in the $\hat{z}$ plane, must be a stagnation point of the cylinder. Using Eq. (19) to evaluate $W\left(\zeta=\zeta_{T E}\right)=0$, we find that for multiple vortices

$$
\Gamma_{\mathrm{b}}=\Gamma_{0}-\sum \kappa_{i} \Gamma_{i},
$$

where

$$
\kappa_{i} \equiv \frac{1-\frac{\rho_{i}}{R} \cos \left(\beta+\tau_{i}\right)}{\frac{1}{2}\left[\left(\frac{\rho_{i}}{R}\right)^{2}+1\right]-\frac{\rho_{i}}{R} \cos \left(\beta+\tau_{i}\right)},
$$

is a geometric coefficient that takes into account the relative position of the $i$-th LEV with respect to the arc. For the vortices that are on the arc, $\rho_{i}=R$ and $\kappa_{i}=1$.

Using the Kutta-Joukowski theorem, the lift coefficient for the circular arc in the presence of the LEV is written as

$$
C_{L}=-\frac{\Gamma_{\mathrm{b}}+\Gamma_{\mathrm{LEV}}}{\frac{1}{2} U_{\infty} c}=-\frac{\Gamma_{\mathrm{b}}}{\frac{1}{2} U_{\infty} c}-\frac{\Gamma_{\mathrm{LEV}}}{\frac{1}{2} U_{\infty} c} .
$$

In the Results, the lift coefficient contribution due to the bound circulation

$$
C_{L_{\mathrm{b}}} \equiv-\frac{\Gamma_{\mathrm{b}}}{\frac{1}{2} U_{\infty} c}
$$

and the lift coefficient contribution due to the LEV

$$
C_{L_{\mathrm{LEV}}} \equiv-\frac{\Gamma_{\mathrm{LEV}}}{\frac{1}{2} U_{\infty} c}
$$

will be compared.

It is important to recall that this is a two-dimensional model that only applies to the particular PIV measurement plane that is being analysed. With this approach we neglect the out-of-plane momentum fluxes, the effect of viscosity (including trailing edge separation) and unsteady effects. The model accounts only for the strongest nuclei of vorticity included in the PIV window, as well as their mirror vortices. Once the vortices have been shed downstream, these are no longer included, nor are their image vortices. For Kelvin's theorem, the circulation shed downstream with an LEV must be balanced by an equal production and shedding of counter-rotating vorticity from the trailing edge. As long as the bound circulation remains almost constant, as in the tested conditions, the net vorticity shed in the wake must be small. Here we assume that the main contribution to the effective bound circulation is due to the strong nuclei of vorticity near the arc, and thus we neglect the contribution of the shed leading-edge and trailing-edge vorticity. Therefore, the lift that is obtained with the model is a first order estimate of the cross-sectional lift.

\subsection{Computation of Vortex Circulation}

The circulation of the vortex was computed by integration of the velocity over the iso-contours of (Eq. 15). Low $\gamma_{2}$ values resulted in large integration paths with 

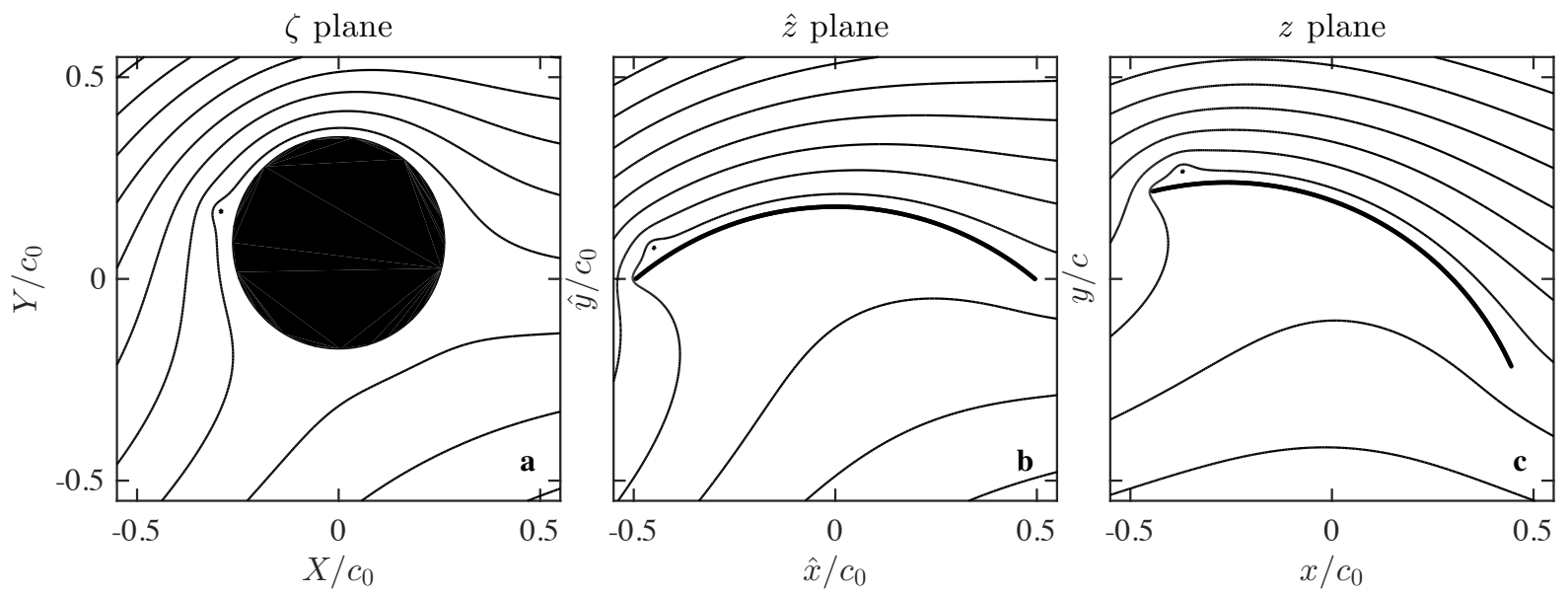

Figure 8: Velocity potential streamlines in the $\zeta$ plane (a) and the transformations in Eq. 11 (b) and Eq. 12 (c)

unclear distinction between the different nuclei of vorticity. On the other hand, high $\gamma_{2}$ values do not allow capturing some of the vortices. A clear description of the vorticity field is achieved when $\gamma_{2}$ ranges between 0.6 and 0.8 . On average, the maximum value of the circulation is achieved with $\gamma_{2} \approx 0.67$. This is consistent with the values adopted by Pitt Ford and Babinsky (2013) and by Eslam Panah et al. (2015).

As shown in Fig. 6, $\gamma_{2}=0.67$ corresponds to a circular contour with $r / l_{o}=1.1$ around an ideal LambOseen vortex. However, to include all of the vorticity of the vortex, it should be necessarily to integrate over a $\gamma_{2}=0.20$ that corresponds to $r / l_{o}=2.5$. Because the contour $\gamma_{2}=0.20$ was not available, a methodology similar to that proposed by Morgan et al. (2009) was followed to estimate the circulation left outside of the iso-contour $\gamma_{2}=0.67$. The circulation was computed integrating over different $\gamma_{2}$ contours between 0.6 and 0.8 , and then these circulations were fitted with those of an ideal Lamb-Oseen vortex (Eq. 5). The fitting, which was performed with a least square method, allowed to identify the total circulation of the vortex. The total circulation was found to be $35 \%$ higher than that computed with $\gamma_{2}=0.67$. Therefore, the integrated circulation of each vortex was multiplied by 1.35 .

\section{Results}

\subsection{Velocity and Vorticity Fields}

Figure 9 shows the time-averaged vector fields, streamlines and vorticity contours for the planes A, B and $\mathrm{C}$. The local angle of attack increases from plane A to plane $\mathrm{C}$ due to the twist of the sail. The maximum camber also increases from plane A to plane C. Planes $\mathrm{A}$ and $\mathrm{B}$ show flow separation at the leading edge and flow reattachment further downstream. The concentric streamlines of Fig. 9e show the averaged flow field of a leading-edge vortex. The streamlines are concentric because part of the onset flow is trapped inside the vortex core and ejected out of the plane of the figure toward the head of the sail. As showed in the following section (3.2), some of the vorticity which is generated at the sharp leading edge, is convected inside a vortex that is stably attached to the leading edge. The permanent and three-dimensional nature of this flow structure make it more similar to the LEV of a delta wing, rather than the laminar separation bubble, which is defined in a timeaveraged sense, of a thin foil at transitional Reynolds numbers.

On plane $\mathrm{C}$, the flow remains attached at the leading edge but separates at $x / c=-0.3$ without reattaching. Vorticity contours show the shear layer generated at the leading edge for the three planes. The separated shear layer curves down in planes A (Fig. 9g) and B (Fig. 9h) showing the effect of the high circulation on these planes, while it is straighter on plane C (Fig. 9i) where trailing edge separation occurs.

The LEV is formed in the upper part of the sail (plane B) and grows in size towards the tip. The growth of the vortex is the result of the vorticity that, at every section, is convected in the axis of the vortex and that, hence, accumulates towards the tip. From plane B to plane A, it can be observed that the axis of the vortex moves away from the sail surface and inboard. 


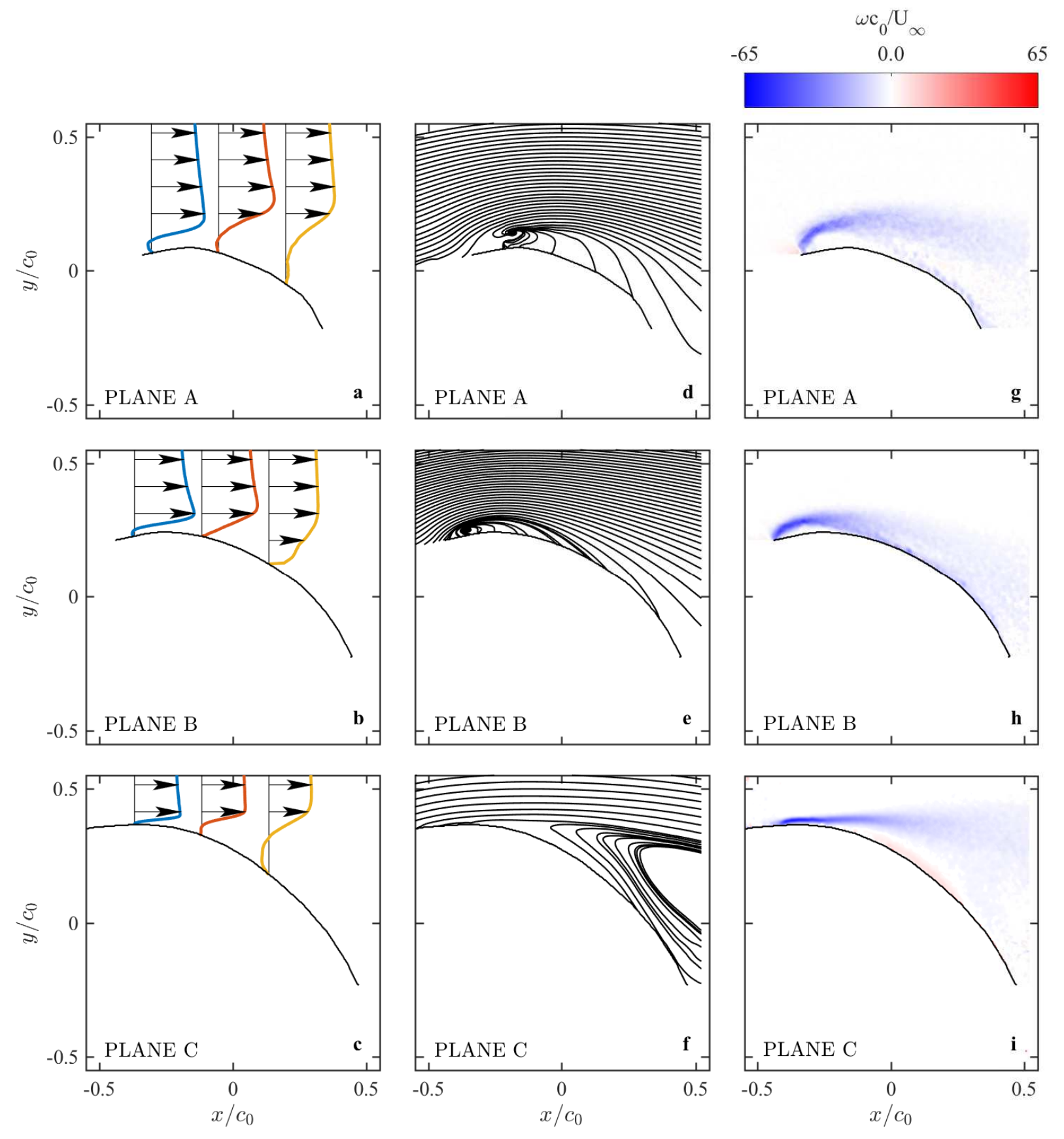

Figure 9: Time-averaged velocity profiles $(\mathbf{a}, \mathbf{b}, \mathbf{c})$, streamlines $(\mathbf{d}, \mathbf{e}, \mathbf{f})$ and contours of non-dimensional vorticity $(\mathbf{g}$, $\mathbf{h}, \mathbf{i}$ ) on the planes A, B and C. Data is averaged over a period $\Delta t^{*}=40.736$, in the interval $1<i<305$, where $i$ is the number of the PIV image pair

\subsection{Unsteady Flow}

The vortex dynamics is investigated using a data set of 305 pair of images sampled every $t^{*}=0.134$, where time $t$ is made non-dimensional with the chordwise convection period $c_{0} / U_{\infty}$, i.e. $t^{*} \equiv t U_{\infty} / c_{0}$. Consider $i$ the index of the pair of images, where $1<i<305$. Figure 


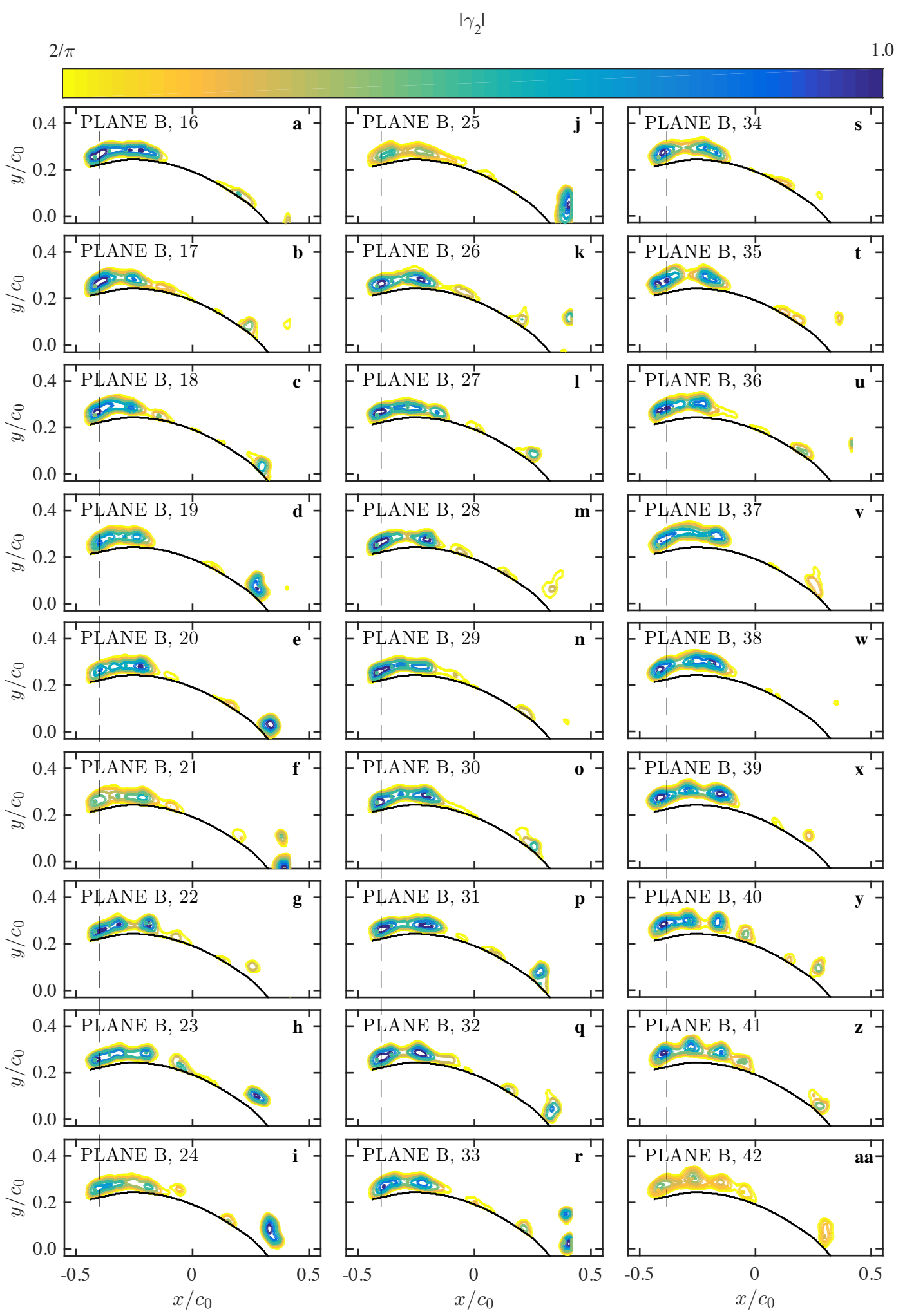

Figure 10: $\gamma_{2}$ criterion on instantaneous velocity fields for plane B (a-aa). Data corresponds to a period of $\Delta t^{*}=3.484$, in the interval $16<i<42$ 


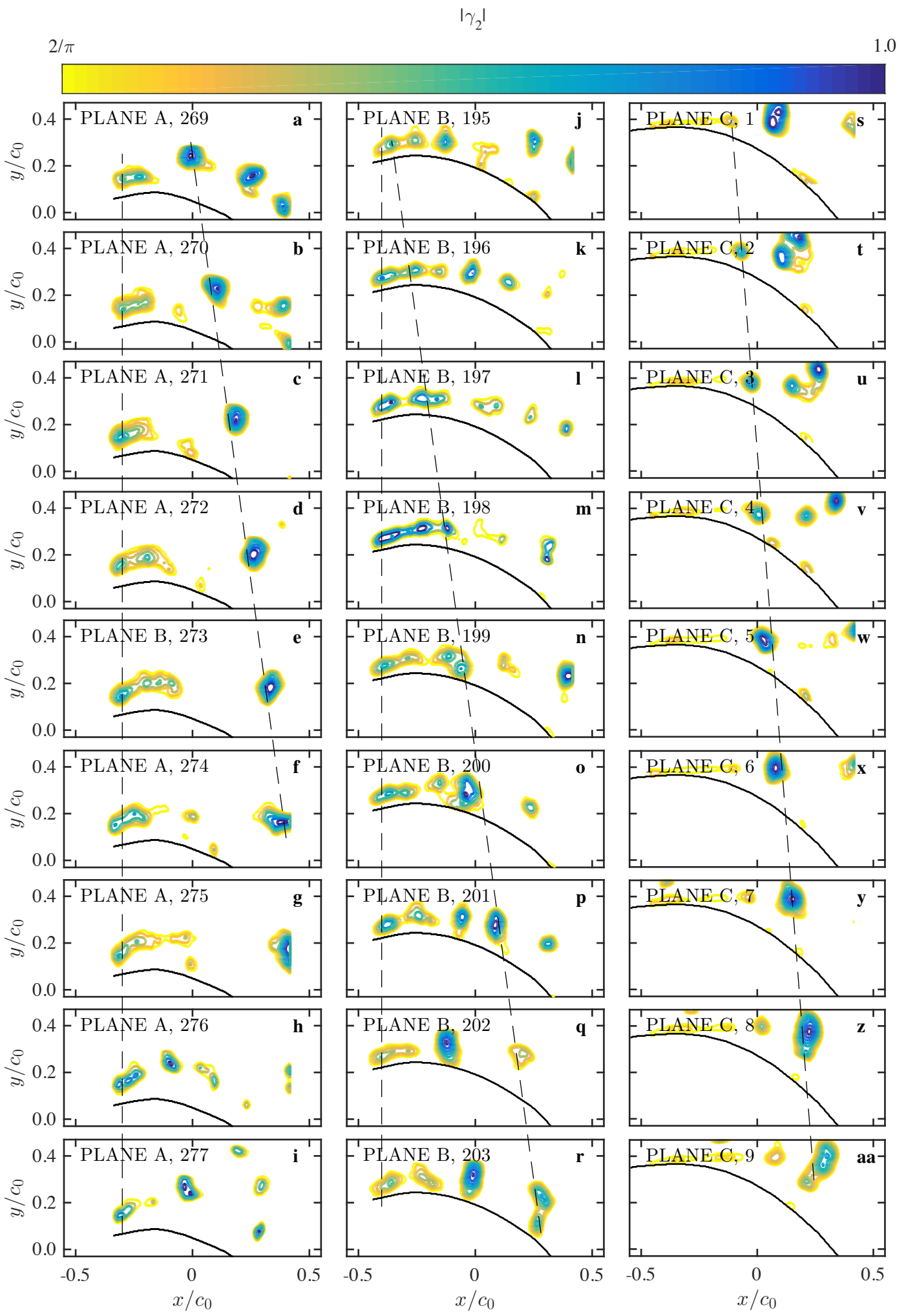

Figure 11: $\gamma_{2}$ criterion on instantaneous velocity fields showing vortex shedding for planes A (a-i), B (j-r) and C (s-aa). Data corresponds to a period of $\Delta t^{*}=1.072$, in the intervals $269<i<277,195<i<203$ and $1<i<9$ for planes A, B and C, respectively 

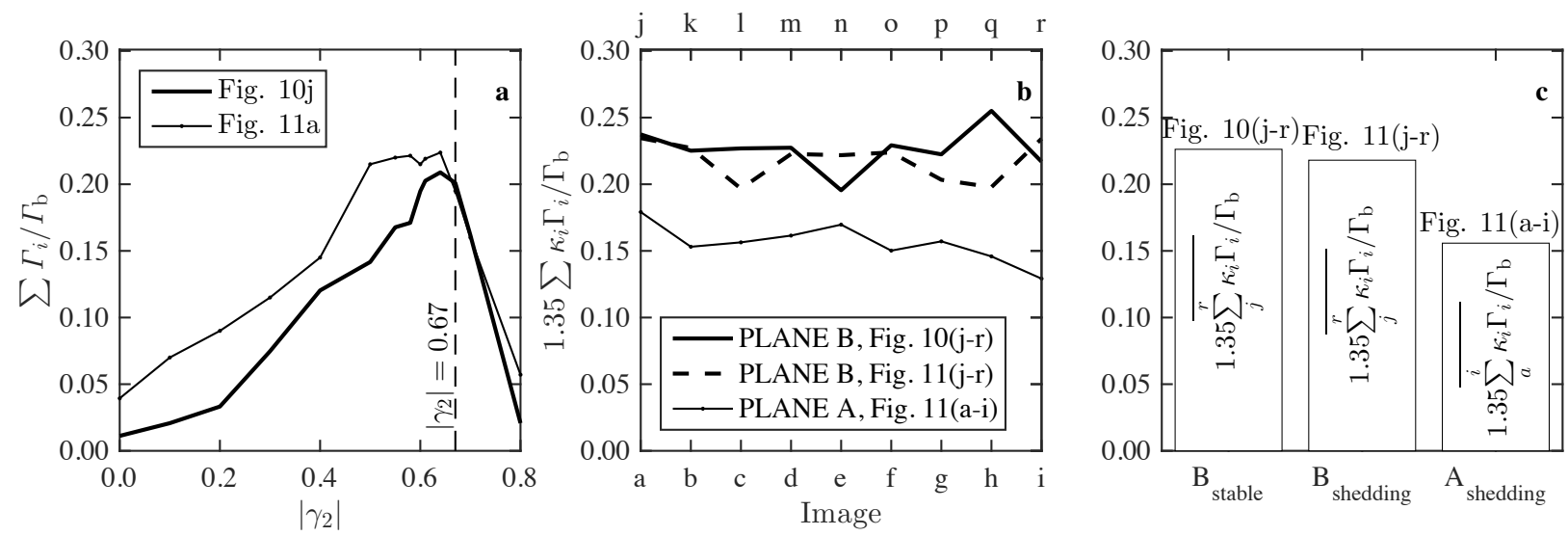

Figure 12: a Sum of circulation of free vortices $\sum \Gamma_{i} / \Gamma_{\mathrm{b}}$ from Fig. 10j and Fig. 11a calculated with different $\gamma_{2}$ isocontour values. b Instantaneous sum of circulation $\sum \kappa_{i} \Gamma_{i} / \Gamma_{\mathrm{b}}$ (adjusted by the correction factor 1.35) of the free vortices in Fig. 10(j-r), 11(j-r) and Fig. 11(a-i), integrated along $\left|\gamma_{2}\right|=0.67$. c Average circulation for the three cases presented in Fig. 12b

10 shows the $\gamma_{2}$ contours for the instantaneous velocity fields on plane B for a period $\Delta t^{*}=3.484$ within the interval $16<i<42$. A stable $\mathrm{LEV}$, which remains attached to the leading edge, can be observed throughout this sequence. The incipiency of vortex shedding can be observed towards the end of the sequence. The LEV on this plane is, in fact, intermittently stable. In particular, it is stable for the sampling periods $1<i<65$, $115<i<170$ and $264<i<305$.

Examples of shedding LEV are presented in Fig. 11 on the planes A, B and C. Each sequence has a time length $\Delta t^{*}=1.072$, but the sequences are taken at different times on each plane, since the experimental setup does not allow simultaneous recording of the planes. It can be observed that the LEV is not stable and it is shed downstream with a convective velocity of approximately $0.6 U_{\infty}$ in plane $\mathrm{A}$ and $\mathrm{B}$, and $0.3 U_{\infty}$ in plane C.

The vortical structures observed in Fig. 10 and 11 are the results of Kelvin-Helmholtz instabilities of the vortex sheet generated at the leading edge. This flow field resemble the separated-reattached flow downstream of sharp obstacles (Bradshaw and Wong, 1972) and blunt flat plates (Kiya and Sasaki, 1983; Bradshaw and Wong, 1972; Ota et al., 1981; Stevenson et al., 2016). The coexistence of a stationary and unsteady vortices is also observed in other vortex dominated flows such as, for example, on delta wings. Gad-El-Hak and Blackwelder (1985) were the first to observed experimentally nuclei of unsteady vorticity coexisting with the primary LEV on delta wings. In a successive work, (Gad-El-Hak and Blackwelder, 1987), the same authors recognised that these unsteady vortices were due to Kelvin-Helmholtz instabilities of the shear layer. Small vorticity concentrations were also observed on non-slender delta wings (Yaniktepe and Rockwell, 2004; Gursul et al., 2005; Muir et al., 2017), which have a swept angle more similar to that of a sail. The averaged vorticity of non-slender delta wings shows a dual-vortex substructure system that resemble the elongated vortex structure near the leading edge of the sail (Fig. 10). Yaniktepe and Rockwell (2004) observed that these nuclei of vorticity could be associated to either jitter of the nominally stationary LEV or to instabilities of the shear layer separating from the leading edge.

Consistently with the observations made in Sec.3.1 on the time averaged flow field, the stationary vortex system near the leading edge in plane A is larger, more distant from the sail surface and more inboard than in plane B.

\subsection{Contribution of the Vortex Circulation to the Total Circulation}

The underlying question that this work aims to address, is the effective contribution of the LEV to the sail performance. Recalling that the total lift coefficient can be broken down into the contribution of the bound circulation $C_{L_{\mathrm{b}}}$ and the contribution of the vortex circulation $C_{L_{\mathrm{LEV}}}$, the ratio $C_{L_{\mathrm{LEV}}} / C_{L_{\mathrm{b}}}=\Gamma_{\mathrm{LEV}} / \Gamma_{\mathrm{b}}$ is computed. A first conservative estimate is made that the Kutta condition is satisfied when the LEV is present. Since trailing 
edge separation actually occurs and the Kutta condition is not satisfied, the computed bound circulation is higher than the real value and thus the computed relative contribution of the LEV is conservative.

The coefficient $\kappa$ in Eq. 21 takes into account the distance of the vortices from the sail. Therefore, the total contribution to the sail's circulation provided by the vortices is $\sum \kappa_{i} \Gamma_{i}$.

Figure $12 \mathrm{~b}$ shows how this varies across a sequence of image pairs. Three examples are presented: the stable LEV on plane B in Fig. 10(j-r), the shedding LEV on the same plane in Fig. 11(j-r), and the shedding LEV on plane A in Fig. 11(a-i). More results are included in the supplementary data available in the Edinburgh digital repository (datashare.is.ed.ac.uk). In most of the cases examined, the total contribution of the vortex circulation to the sail's circulation is between $10 \%$ and $20 \%$ of the bound circulation. At every instant, the contribution of the vortex circulation is higher in $\mathrm{B}$ than in plane $\mathrm{A}$, and the difference between the planes is larger than the variations between different instants on the same plane.

The time-averaged contribution of the vortex circulation $\left(\overline{\sum_{a, j}^{i, r} \kappa_{i}} \Gamma_{i}\right)$ is presented in Fig. 12c for the same cases as in Fig. 12b. Here it is clear that the presence of a stable LEV, as opposed to a series of shedding vortices, provides only a marginally higher averaged circulation. It must be remembered, however, that increasing the vortex circulation in the front of the sail, as with a stable LEV, rather than on the rear, would lead to a higher driving force. This effect, however, cannot be assessed with the present analysis.

\section{Conclusions}

Recent high-fidelity numerical simulations (Viola et al., 2014) suggested that a LEV is formed at the leading edge of asymmetric spinnakers and that it remains stably attached to the sail providing lift augmentation. This finding is investigated in the present paper. A model-scale asymmetric spinnaker is tested in uniform flow in a water flume at a Reynolds number of $U_{\infty}$ is $1.3 \times 10^{4}$. The time-averaged velocity field measured with PIV confirms the numerical results. On the highest half of the sail, the LEV is formed and it grows in size towards the head of the sail, while its axis moves away from the sail surface and inboard of the sail. Downstream of the LEV, the time-averaged flow reattaches and an attached boundary layer is formed. Conversely, on the lowest half of the sail, where trailing edge separation is dominant, the LEV is absent or of negligible dimensions and the separation point is well upstream of the mid-chord.

The time-resolved flow field reveals that the LEV remains attached to the leading edge intermittently and only on a section at $3 / 4^{\text {th }}$ of the sail span. For a period of time of the order of $10 c / U_{\infty}$, the LEV is stably attached, and then for a period of similar length the LEV is shed and convects downstream at $60 \%$ of the free stream velocity. The contribution to the sail sectional lift of the vortices is higher than $15 \%-25 \%$, where the estimation is very conservative because a Kutta condition has been assumed to compute the bound circulation. These results suggest that the performance of asymmetric spinnakers could be enhanced significantly by controlling the formation and stability of the LEV.

\section{Acknowledgements}

This work was funded by the Consejo Nacional de Ciencia y Tecnología (CONACYT).

\section{References}

Bethwaite, F., 1993. High Performance Sailing. Waterline Books, UK.

Borazjani, I., Daghooghi, M., 2013. The fish tail motion forms an attached leading edge vortex. Proc R Soc B 280, 20122071. doi:10.1098/rspb. 2012.2071.

Bot, P., Viola, I.M., Flay, R.G.J., Brett, J.S., 2014. Wind-tunnel pressure measurements on model-scale rigid downwind sails. Ocean Eng 90, 84-92. doi:10.1016/j . oceaneng. 2014.07.024.

Bradshaw, P., Wong, F.Y.F., 1972. The reattachment and relaxation of a turbulent shear layer. Journal of Fluid Mechanics 52, 113135. doi:10.1017/S002211207200299X.

Corke, T.C., Thomas, F.O., 2015. Dynamic stall in pitching airfoils: Aerodynamic damping and compressibility effects. Annu Rev Fluid Mech 47, 479-505. doi:10.1146/annurev-fluid-010814-013632.

Ellington, C.P., 1999. The novel aerodynamics of insect flight: applications to micro-air vehicles. J Exp Biol 202, 3439-3448.

Eslam Panah, A., Akkala, J.M., Buchholz, J.H.J., 2015. Vorticity transport and the leading-edge vortex of a plunging airfoil. Experiments in Fluids 56, 160. doi:10.1007/s00348-015-2014-7.

Gad-El-Hak, M., Blackwelder, R.F., 1985. The discrete vortices from a delta wing. AIAA Journal 23, 961-962. URL: https://doi.org/10.2514/3.9016, doi:10.2514/3.9016.

Gad-El-Hak, M., Blackwelder, R.F., 1987. Control of the discrete vortices from a delta wing. AIAA Journal 25, 1042-1049. URL: https://doi.org/10.2514/3.9740, doi:10.2514/3.9740.

Garmann, D.J., Visbal, M.R., Orkwis, P.D., 2013. Three-dimensional flow structure and aerodynamic loading on a revolving wing. Phys Fluids 25. doi:10.1063/1.4794753.

Gordnier, R.E., Visbal, M.R., Gursul, I., Wang, Z., 2009. Computational and experimental investigation of a nonslender delta wing. AIAA J 47, 1811-1825. doi:10.2514/1.37848.

Graftieaux, L., Michard, M., Grosjean, N., 2001. Combining PIV, POD and vortex identification algorithms for the study of unsteady turbulent swirling flows. Meas Sci Technol 12, 1422-1429. doi:10.1088/0957-0233/12/9/307. 
Gursul, I., Gordnier, R., Visbal, M., 2005. Unsteady aerodynamics of nonslender delta wings. Prog Aerosp Sci 41, 515-557. doi:10.1016/j.paerosci.2005.09.002.

Gursul, I., Wang, Z., Vardaki, E., 2007. Review of flow control mechanisms of leading-edge vortices. Prog Aerosp Sci 43, 246-270. doi:10.1016/j.paerosci.2007.08.001.

Harbig, R.R., Sheridan, J., Thompson, M.C., 2013. Reynolds number and aspect ratio effects on the leading-edge vortex for rotating insect wing planforms. J Fluid Mech 717, 166-192. doi:10.1017/jfm. 2012.565.

Hubel, T.Y., Tropea, C., 2010. The importance of leading edge vortices under simplified flapping flight conditions at the size scale of birds. J Exp Biol 213, 1930-1939. doi:10.1242/jeb.047886

Jardin, T., David, L., 2014. Spanwise gradients in flow speed help stabilize leading-edge vortices on revolving wings. Phys Rev E 90, 013011. doi:10.1103/PhysRevE.90.013011.

Kiya, M., Sasaki, K., 1983. Structure of a turbulent separation bubble. Journal of Fluid Mechanics 137, 83113 doi:10.1017/S002211208300230X.

Larsen, J.W., Nielsen, S.R.K., Krenk, S., 2007. Dynamic stall model for wind turbine airfoils. J Fluid Struct 23, 959-982. doi:10.1016/j.jfluidstructs.2007.02.005.

Lentink, D., Dickson, W.B., van Leeuwen, J.L., Dickinson, M.H., 2009. Leading-edge vortices elevate lift of autorotating plant seeds. Science 324, 1438-1440. doi:10.1126/science.1174196.

Lentink, D., Müller, U.K., Stamhuis, E.J., de Kat, R., van Gestel, W., Veldhuis, L.L.M., Henningsson, P., Hedenström, A., Videler, J.J., van Leeuwen, J.L., 2007. How swifts control their glide performance with morphing wings. Nature 446, 1082-1085. doi:10.1038/nature05733.

McWilliams, J.C., 1984. The emergence of isolated coherent vortices in turbulent flow. Journal of Fluid Mechanics 146, 2143. doi:10.1017/S0022112084001750.

Morgan, C.E., Babinsky, H., Harvey, J.K., 2009. Vortex detection methods for use with PIV and CFD data, in: Proceedings of the 47th AIAA Aerospace Sciences Meeting, Orlando, Florida, USA. doi:10.2514/6.2009-74.

Muijres, F.T., Johansson, L.C., Barfield, R., Wolf, M., Spedding, G.R., Hedenström, A., 2008. Leading-Edge Vortex Improves Lift in Slow-Flying Bats. Science 319, 1250-1253. doi:10.1126/science.1153019.

Muir, R.E., Arredondo-Galeana, A., Viola, I.M., 2017. The leadingedge vortex of swift wing-shaped delta wings. Royal Society Open Science 4. doi:10.1098/rsos. 170077

Nava, S., Cater, J., Norris, S., 2016. A Comparison of RANS and LES for Upwind Sailing Aerodynamics, in: Proceedings of the 22nd Chesapeake Sailing Yacht Symposium, Annapolis, Mryland, USA.

Ota, T., Asano, Y., ichi Okawa, J., 1981. Reattachment length and transition of the separated flow over blunt flat plates. Bulletin of JSME 24, 941-947. doi:10.1299/j sme1958.24.941.

Pitt Ford, C., Babinsky, H., 2013. Lift and the leading-edge vortex. J Fluid Mech 720, 280-313. doi:10.1017/jfm.2013.28.

Rabinovitch, J., Brion, V., Blanquart, G., 2012. Effect of a splitter plate on the dynamics of a vortex pair. Phys Fluids 24. doi:10.1063/1.4737878.

Srygley, R.B., Thomas, A.L.R., 2002. Unconventional lift-generating mechanisms in free-flying butterflies. Nature 420, 660-664. doi:10.1038/nature01223.

Stevenson, J.P., Walsh, E.J., Nolan, K.P., $2016 . \quad$ Visualization of the vortex and reverse-flow structure of a separation bubble. J. Vis. 19, 175-177. URL: http://dx.doi.org/10.1007/s12650-015-0306-x, doi:10.1007/s12650-015-0306-x.

Viola, I.M., Bartesaghi, S., Van-Renterghem, T., Ponzini, R., 2014.
Detached eddy simulation of a sailing yacht. Ocean Eng 90, 93103. doi:10.1016/j.oceaneng.2014.07.019.

Viola, I.M., Bot, P., Riotte, M., 2013. Upwind sail aerodynamics: A RANS numerical investigation validated with wind tunnel pressure measurements. International Journal of Heat and Fluid Flow 39, 90-101. doi:10.1016/j.ijheatfluidflow.2012.10.004.

Viola, I.M., Flay, R.G.J., 2009. Force and pressure investigation of modern asymmetric spinnakers. International Journal of Small Craft Technology Transaction RINA Part B2 151, 31-40. doi:10.3940/rina.ijsct.2009.b2.98.

Viola, I.M., Flay, R.G.J., 2010. Pressure distributions on modern asymmetric spinnakers. International Journal of Small Craft Technology Transaction RINA Part B1 152, 41-50. doi:10.3940/rina.ijsct.2010.b1.103.

Viola, I.M., Flay, R.G.J., 2011a. Sail aerodynamics: Understanding pressure distributions on upwind sails. Exp Therm and Fluid Sci 35, 1497-1504. doi:10.1016/j.expthermflusci.2011.06.009.

Viola, I.M., Flay, R.G.J., 2011b. Sail pressures from full-scale, windtunnel and numerical investigations. Ocean Eng 38, 1733-1743. doi:10.1016/j.oceaneng. 2011.08.001.

Viola, I.M., Flay, R.G.J., 2012. Sail aerodynamics: on-water pressure measurements on a downwind sail. J Ship Res 56, 197-206. doi:10.5957/JOSR.56.4.110003

Viola, I.M., Flay, R.G.J., 2015. Aerodynamics of headsails: a review of measured surface pressures and expected flow fields, in: Proceedings of the 5th High Performance Yacht Design Conference, Auckland, New Zealand.

Wereley, S.T., Gui, L., Meinhart, C.D., 2002. Advanced algorithms for microscale Particle Image Velocimetry. AIAA J 40, 10471055 .

Yaniktepe, B., Rockwell, D., 2004. Flow structure on a delta wing of low sweep angle. AIAA Journal 42, 513-523. URL: https://doi.org/10.2514/1.1207, doi:10.2514/1.1207. 


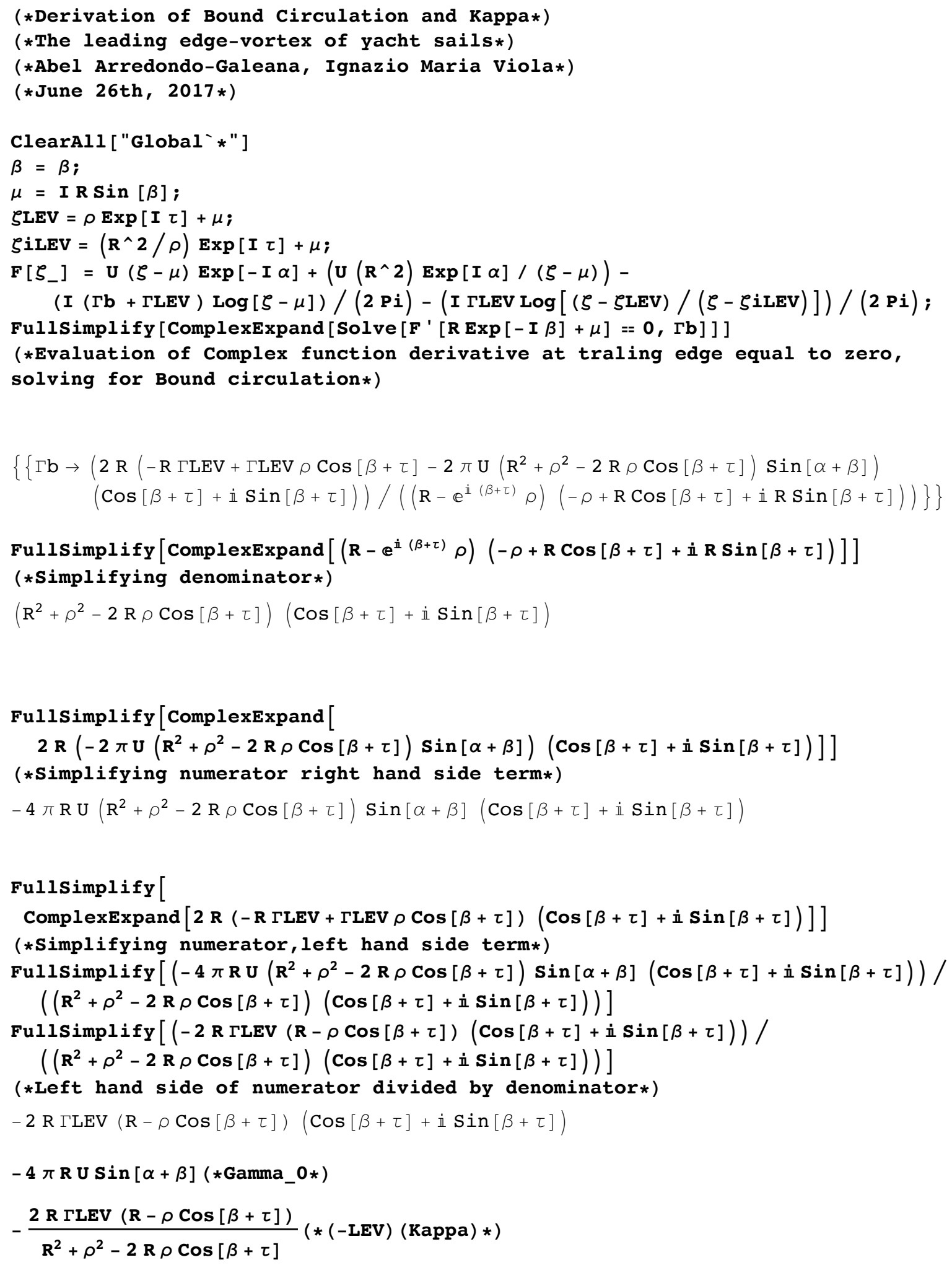


STABLE LEV - PLANE B
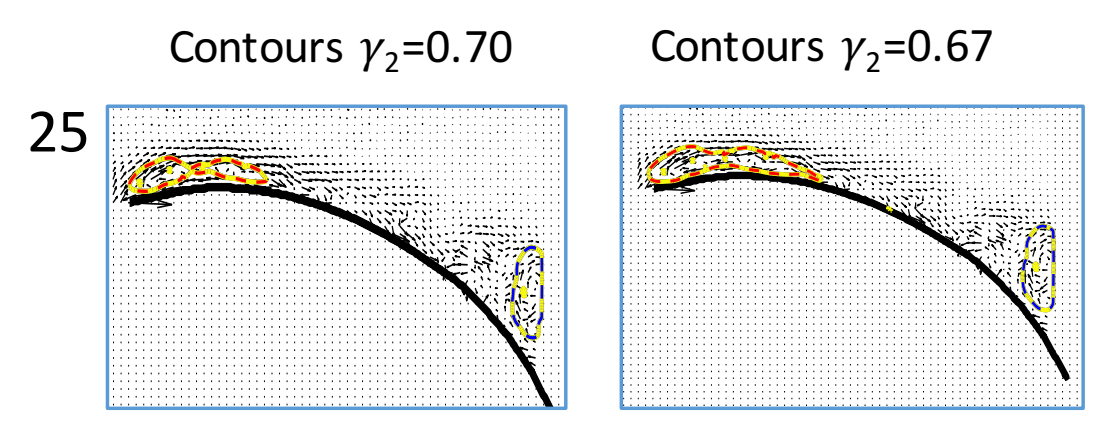

Artificial $\gamma_{2}$ contours

26
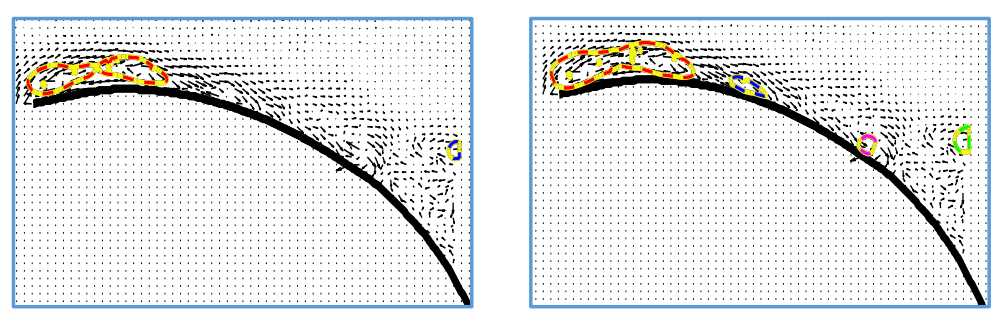

on top of experimental

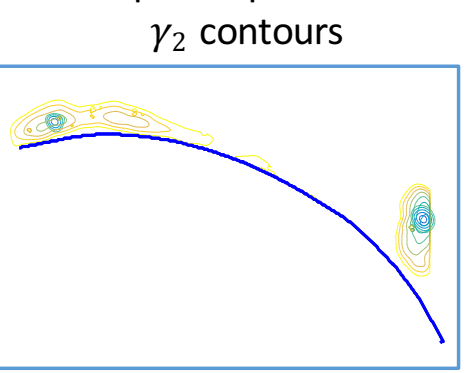

27
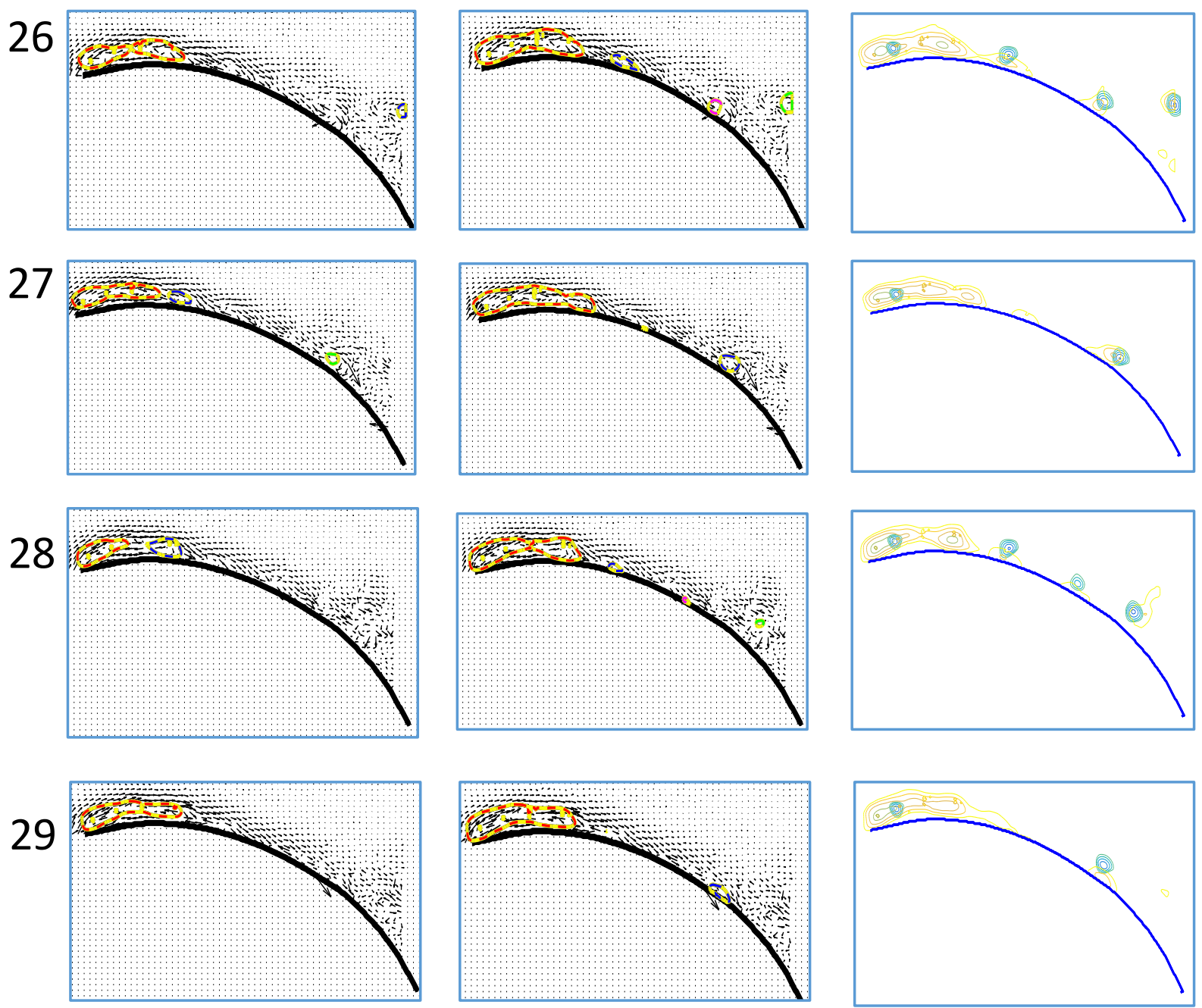

30
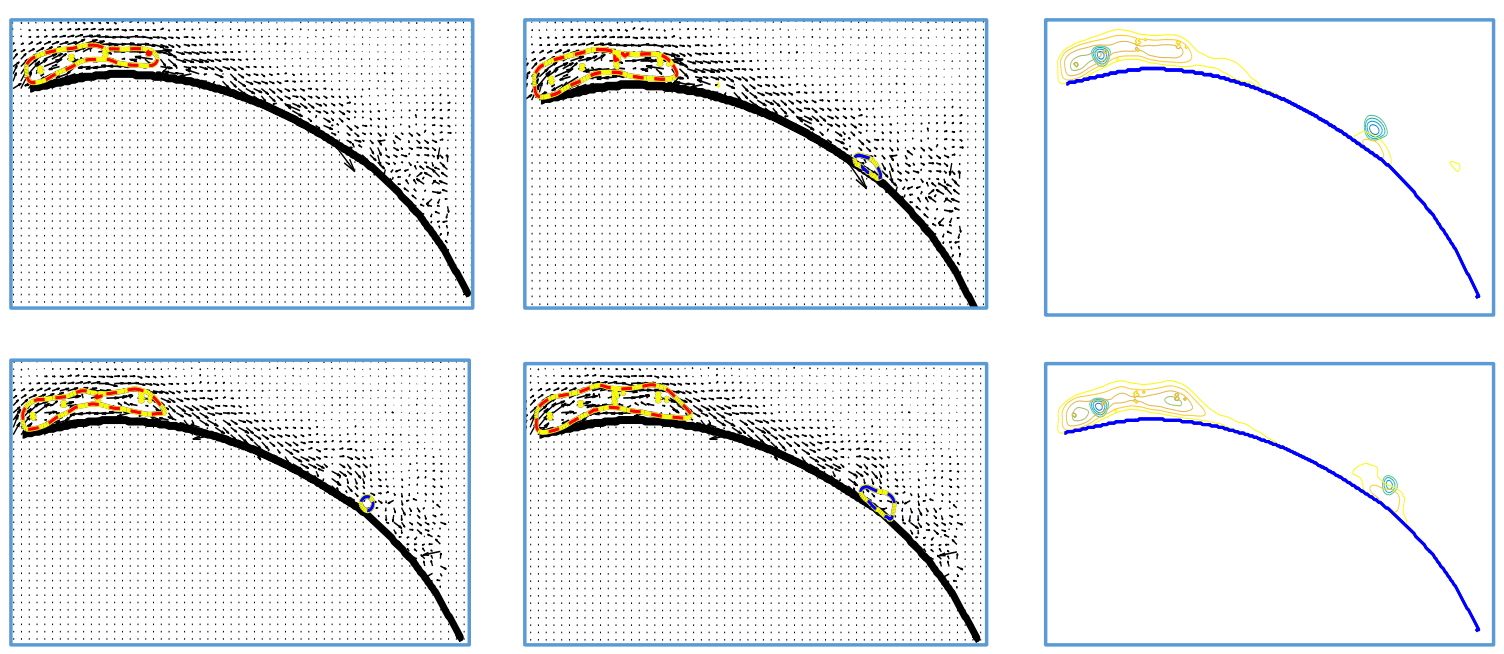
STABLE LEV - PLANE B

\section{Contours $\gamma_{2}=0.70$ \\ Contours $\gamma_{2}=0.67$}

31

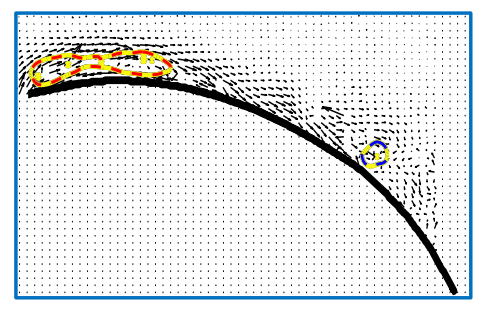

32

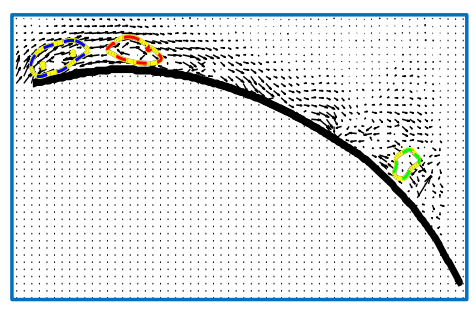

33

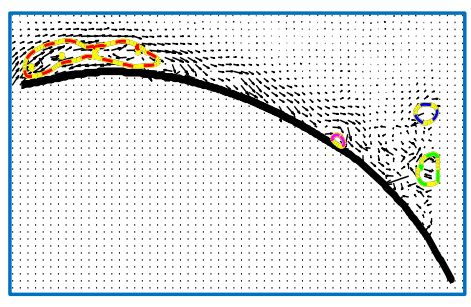

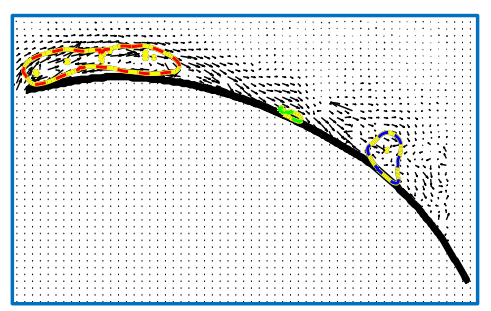
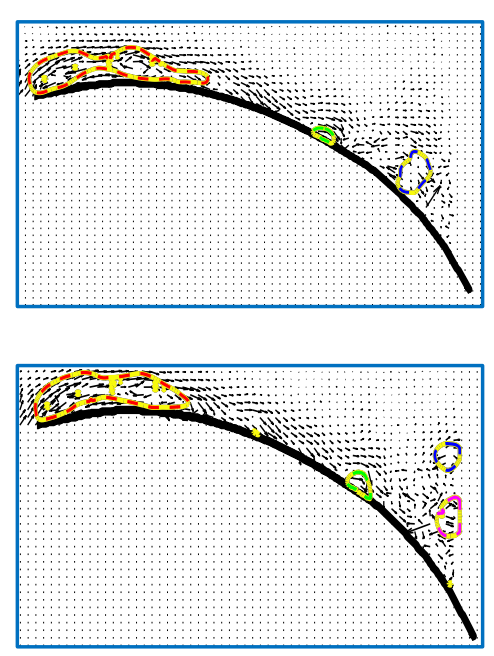

Artificial $\gamma_{2}$ contours on top of experimental $\gamma_{2}$ contours
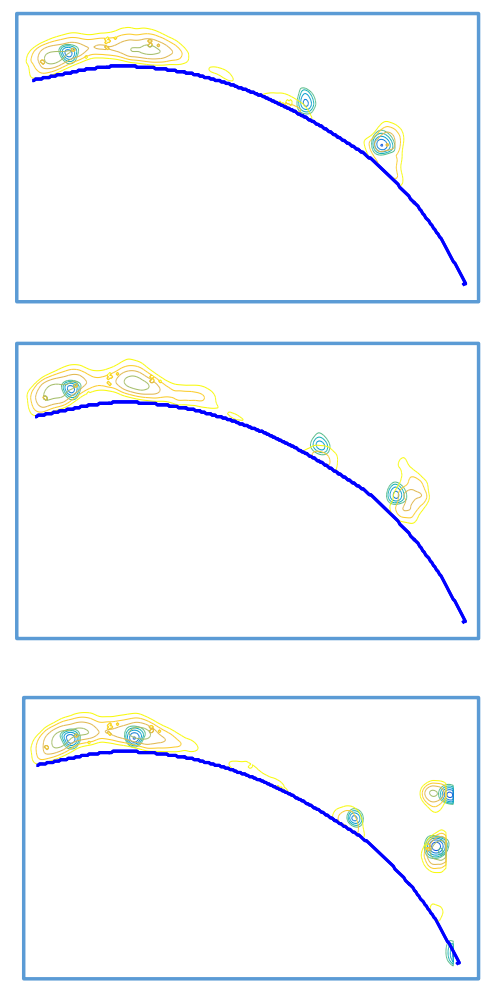


\section{SHEDDING LEV - PLANE B}

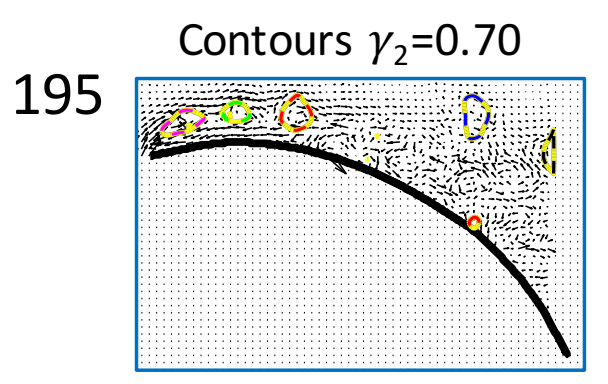

\section{6}

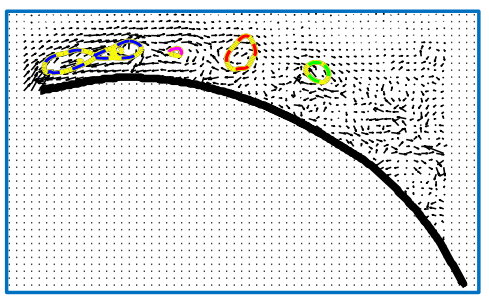

197
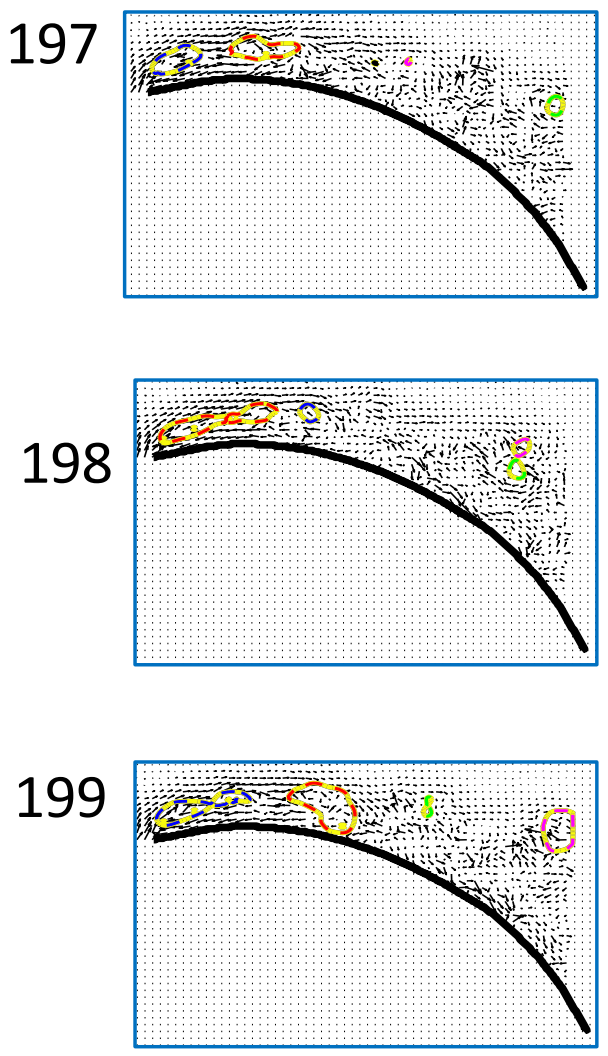

200

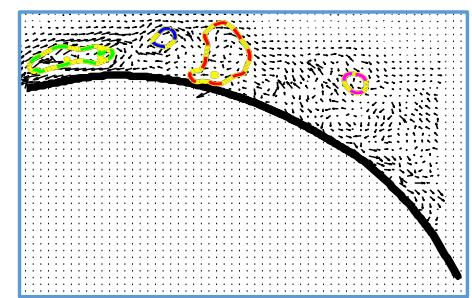

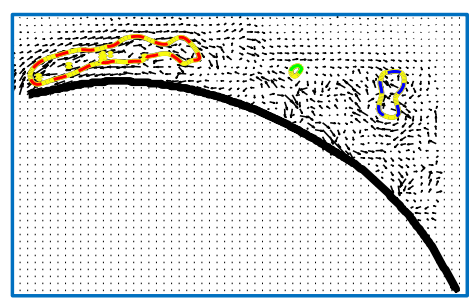
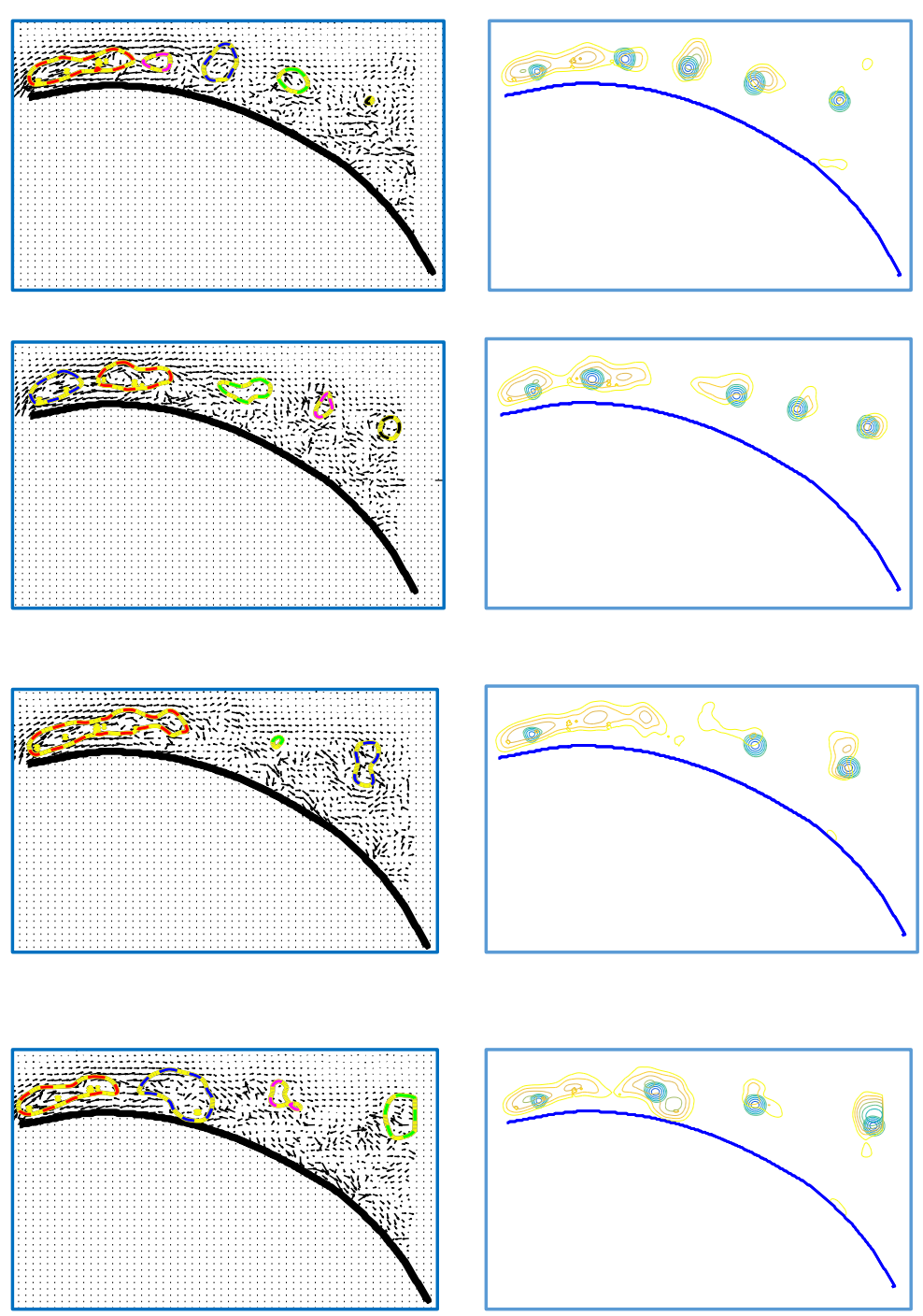

Artificial $\gamma_{2}$ contours on top of experimental $\gamma_{2}$ contours
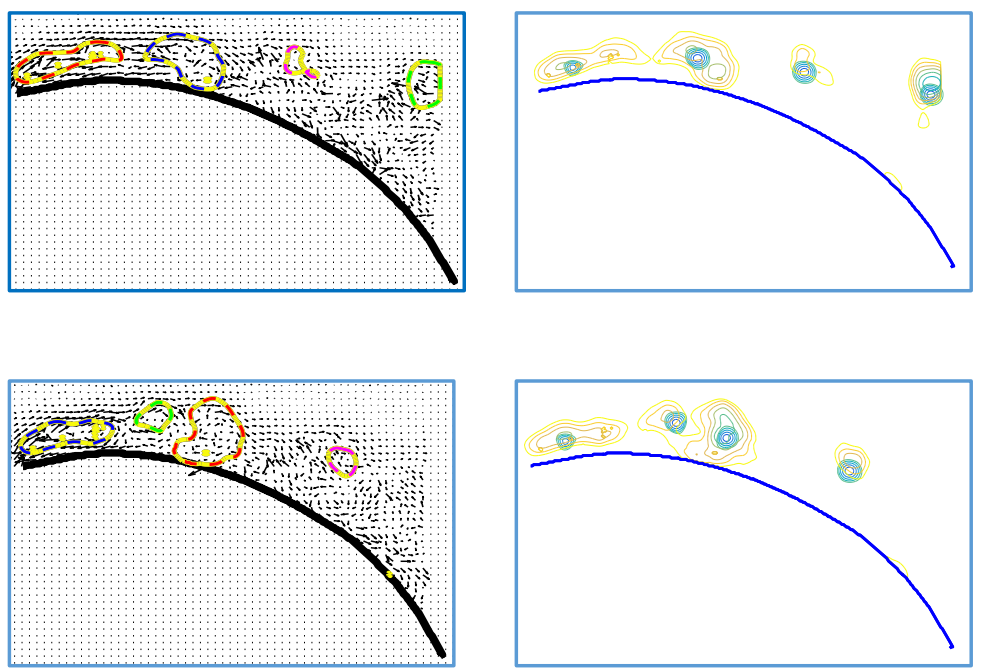

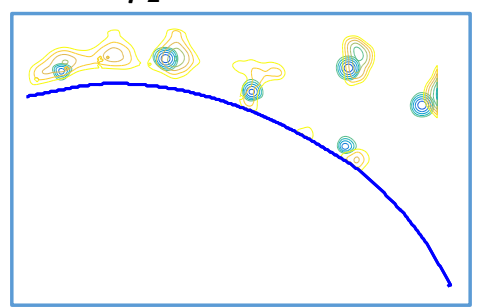




\section{SHEDDING LEV - PLANE B}
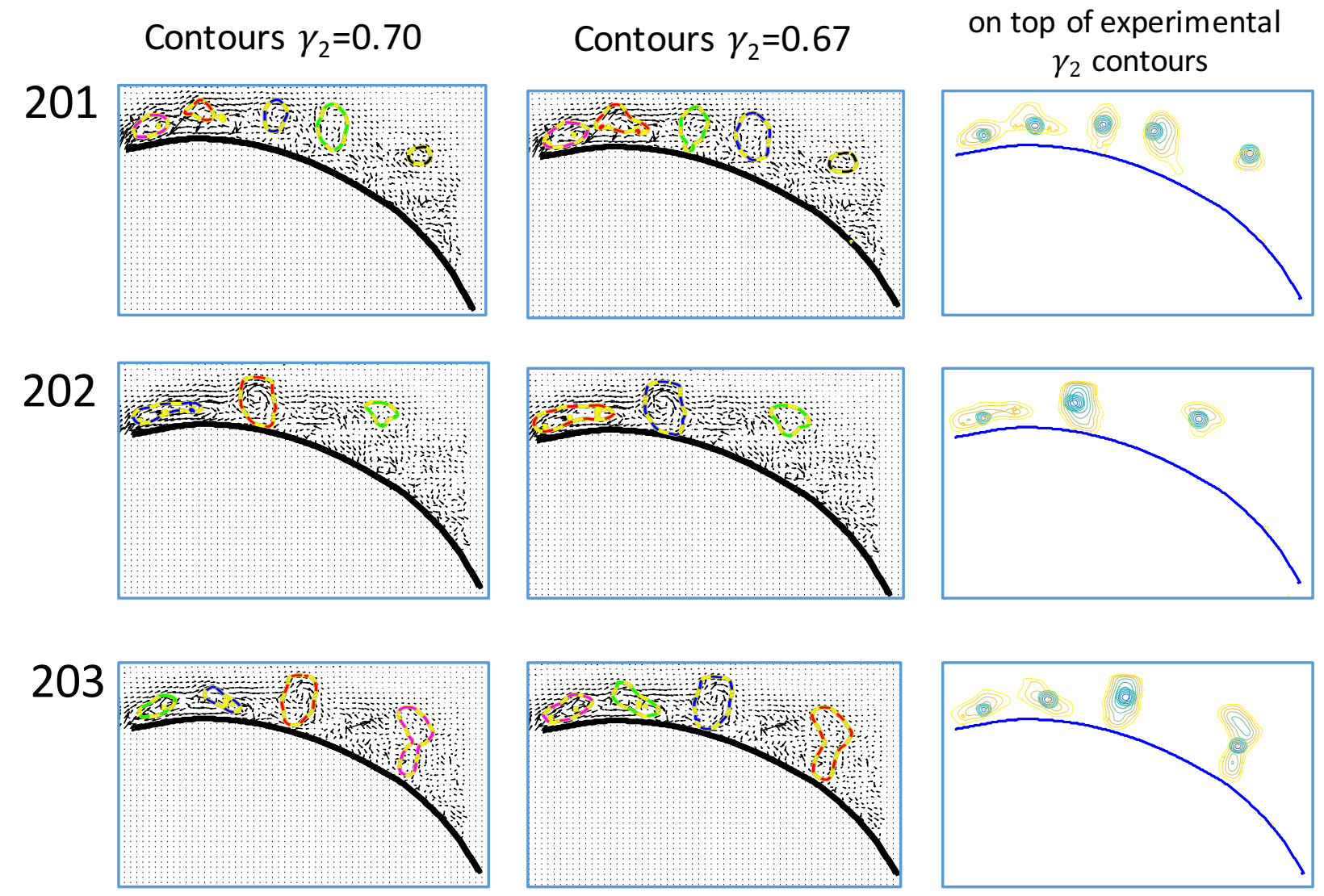


\section{SHEDDING LEV - PLANE A}

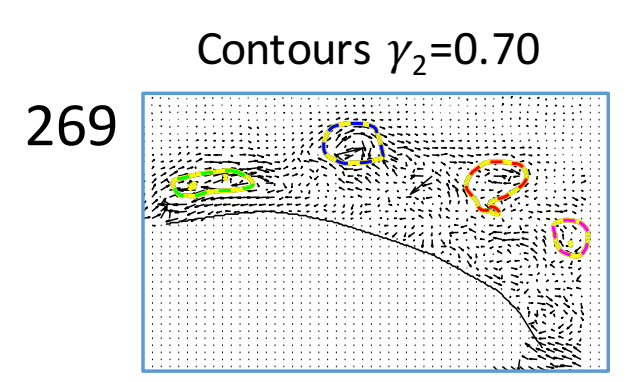

\section{Contours $\gamma_{2}=0.67$}
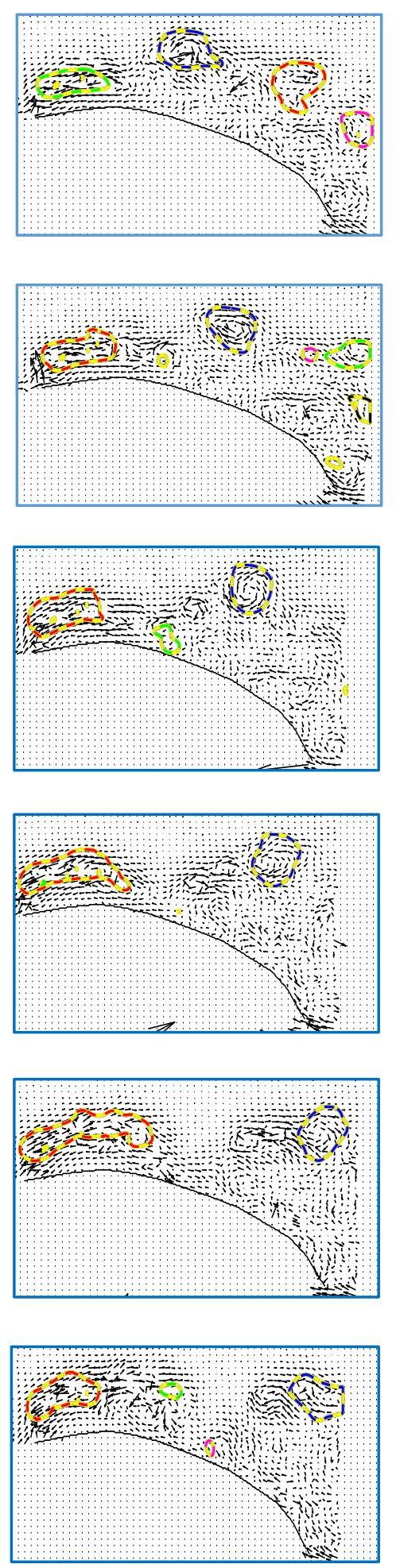

Artificial $\gamma_{2}$ contours on top of experimental $\gamma_{2}$ contours
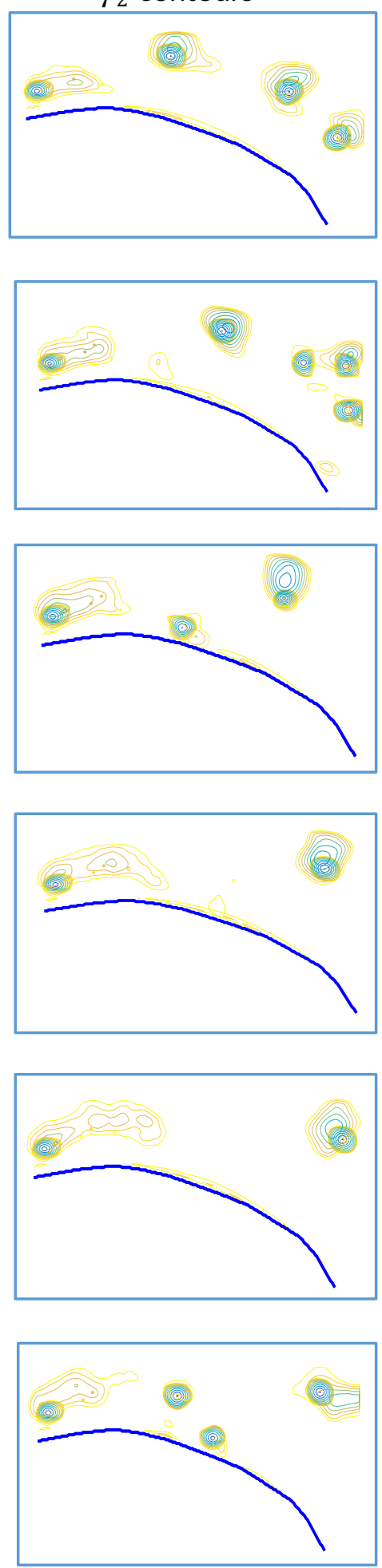


\section{SHEDDING LEV - PLANE A}

Contours $\gamma_{2}=0.70$

275

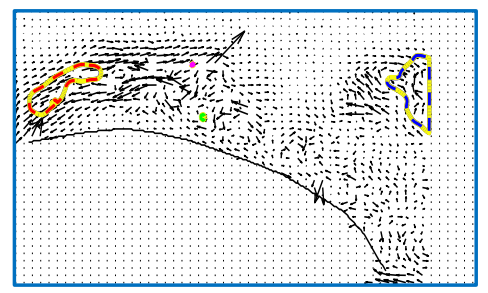

276

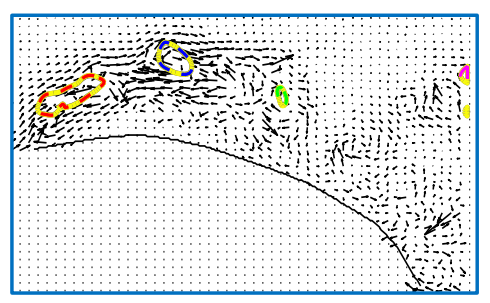

277

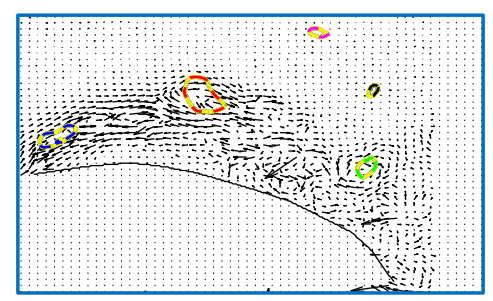

Contours $\gamma_{2}=0.67$

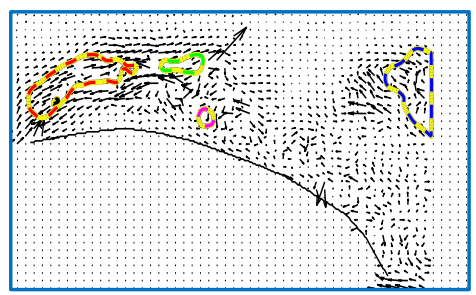

Artificial $\gamma_{2}$ contours on top of experimental $\gamma_{2}$ contours
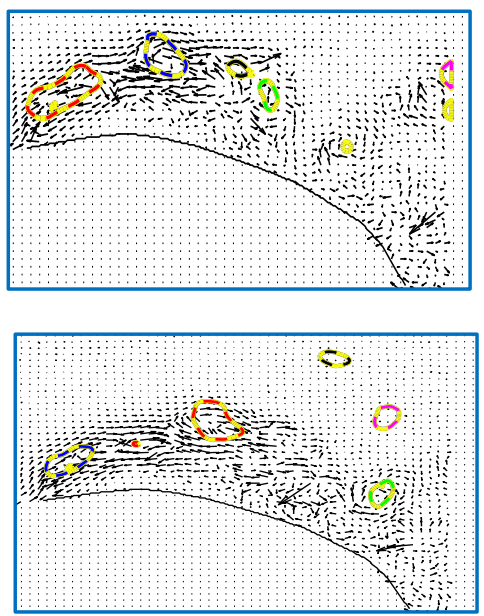
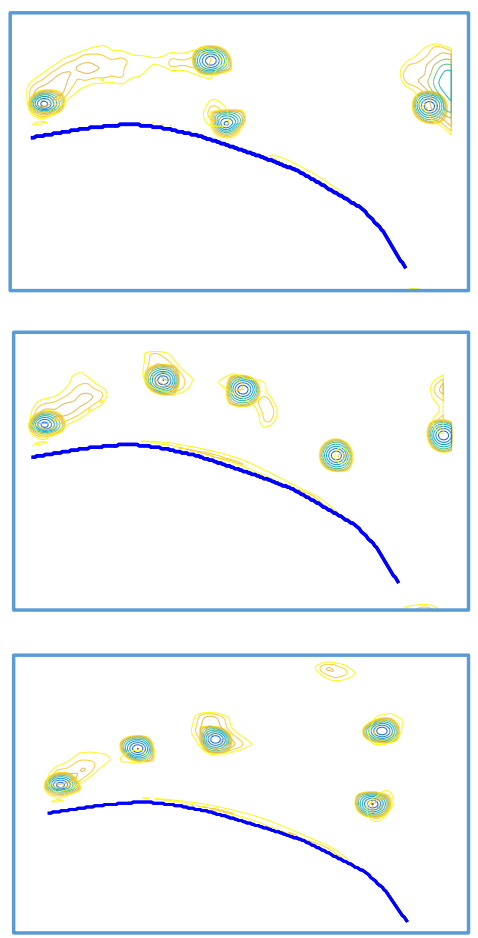
Total circulation plotted in Fig. 12a for different gamma2 thresholds applied to figs. 10j and 11a, correction factor 1.35 for vorticity outside of the core is not applied

\begin{tabular}{|c|c|c|c|c|c|c|c|c|c|c|c|c|c|c|c|c|c|c|c|c|c|c|c|c|}
\hline & 10j & und Circu & & & & & & & & & & & & & & & & & & & & & & \\
\hline Gamma2 & Tao & Rho & k1 & Gamma1 & Tao & Rho & k2 & Gamma2 & Tao & Rho & k3 & Gamma3 & \begin{tabular}{|l|l|} 
Tao \\
\end{tabular} & Rho & \begin{tabular}{l|l|l}
$\mathrm{k} 4$ & $\mathrm{k}$
\end{tabular} & \begin{tabular}{|l|} 
Gamma4 \\
\end{tabular} & k1Gamma1 & k2Gamma2 & k3Gamma3 & \begin{tabular}{|l|l|} 
k4Gamma4 \\
\end{tabular} & Totalkappa & TotalkappaNorm & $\begin{array}{l}\text { Total } \\
\end{array}$ & $\begin{array}{l}\text { TotalNorm } \\
\text { Toln }\end{array}$ \\
\hline 0.8 & 165 & 1.15 & 0.9301 & & & & & & & & & & & 1.35 & 0.5093 & -0.00050086 & & & & & -0.0002551 & \begin{tabular}{|l|}
0.010584564 \\
\end{tabular} & -0.00050086 & 0.020782573 \\
\hline $\begin{array}{l}0.7 \\
0.67 \\
\end{array}$ & $\frac{165}{165}$ & $\begin{array}{l}1.15 \\
1.15\end{array}$ & 0.9301 & $\begin{array}{l}\mid-0.0032 \\
-0.0022 \\
\end{array}$ & & & & & & & & & 45 & $\begin{array}{r}1.35 \\
.25 \\
\end{array}$ & 0.5093 & 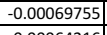 & \begin{tabular}{|l|}
-0.0029763 \\
\end{tabular} & & 0 & \begin{tabular}{|l|l|}
-0.0003553 \\
\end{tabular} & -0.0033316 & $\begin{array}{l}0.138239926 \\
0.176670\end{array}$ & -0.00389755 & 0.161724066 \\
\hline 0.64 & 165 & $\frac{1.15}{1.15}$ & 0.9301 & $\begin{array}{c}-0.00442 \\
-0.0040\end{array}$ & \begin{tabular}{ll|}
1.1 \\
\end{tabular} & 112 & \begin{tabular}{|c|c|c|c|}
0.949 \\
\end{tabular} & 0.000 & & & & & $\frac{45}{45}$ & $\frac{1.35}{1.35}$ & $\begin{array}{l}0.5093 \\
0.5093 \\
\end{array}$ & -0.00064216 & \begin{tabular}{|l|l|}
-0.0039064 \\
\end{tabular} & & & \begin{tabular}{|l|}
-0.0003271 \\
0
\end{tabular} & -0.0042335 & 0.175662742 & $\begin{array}{r}-0.00484216 \\
-0.005324511\end{array}$ & 0.200919502 \\
\hline 0.62 & 165 & 1.15 & $\begin{array}{l}0.9301 \\
\end{array}$ & $|-0.00437|$ & 1.1 & 112 & 0.9429 & 0.000 & & & & & 45 & 1.35 & $\begin{array}{l}0.5093 \\
0.5093 \\
\end{array}$ & $\mid$ & \begin{tabular}{|c|}
-0.00400599 \\
-0.0040
\end{tabular} & & & \begin{tabular}{|l|}
-0.00003212 \\
-0.0001324 \\
\end{tabular} & 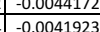 & . & - & $\begin{array}{l}0.20889888 / 3 \\
0.191908465 \\
\end{array}$ \\
\hline 0.61 & 165 & 1.15 & 0.9301 & -0.004200 & 1.1 & 112 & $\begin{array}{ll}0.9429 \\
\end{array}$ & 0.000 & & & & & 45 & 1.35 & 0.5093 & -0.00068223 & \begin{tabular}{|c|}
-0.0039064 \\
\end{tabular} & & & \begin{tabular}{|l|l|}
-0.0003475 \\
\end{tabular} & -0.0042539 & 0.176509533 & -0.00488223 & 0.202582158 \\
\hline 0.6 & 165 & 1.15 & 0.9301 & -0.00400 & \begin{tabular}{ll|}
1.1 \\
\end{tabular} & 112 & 0.9429 & 0.000 & & & & & 45 & 1.35 & 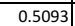 & $\mid-0.0006941$ & $\mid-0.0037204$ & & 0 & $\mid-0.0003535$ & 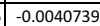 & 0.169041707 & -0.0046941 & 0.194775934 \\
\hline 0.58 & 165 & 1.15 & 0.9301 & -0.00340 & \begin{tabular}{ll|l}
1.1 \\
\end{tabular} & 112 & 0.9429 & 0.000 & & & & & 45 & 1.35 & 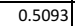 & -0.0007226 & $\mid-0.0031623$ & & 0 & $\mid-0.000368$ & -0.0035304 & 0.146487763 & -0.00412259 & 0.171061826 \\
\hline 0.55 & 165 & 1.15 & 0.9301 & -0.00330 & \begin{tabular}{ll|l} 
& \\
\end{tabular} & 112 & $\begin{array}{l}0.9429 \\
\end{array}$ & 0.000 & & & & & 45 & 1.35 & 0.5093 & -0.0007418 & \begin{tabular}{|l|}
-0.0030693 \\
\end{tabular} & & & \begin{tabular}{|l|}
-0.0003778 \\
\end{tabular} & -0.0034471 & 0.143033542 & -0.00404176 & 0.167707884 \\
\hline 0.5 & 165 & 1.15 & 0.9301 & -0.00280 & 1.1 & 112 & $\begin{array}{ll}0.9429 \\
\end{array}$ & 0.000 & & & & & 45 & 1.35 & $\begin{array}{ll}0.5093 \\
\end{array}$ & -0.0006142 & \begin{tabular}{|l|}
-0.0026043 \\
\end{tabular} & & & \begin{tabular}{|c|}
-0.0003128 \\
\end{tabular} & -0.0029171 & $\begin{array}{l}0.121040953 \\
\end{array}$ & -0.00341419 & 0.141667635 \\
\hline $\begin{array}{ll}0.4 \\
\end{array}$ & 165 & 1.15 & 0.9301 & -0.00290 & 1.1 & 112 & $\begin{array}{ll}0.9429 \\
\end{array}$ & 0.000 & & & & & 45 & 1.35 & $\begin{array}{ll}0.5093 \\
\end{array}$ & 0.0000000 & \begin{tabular}{|l|}
-0.0026973 \\
\end{tabular} & & 0 & & \begin{tabular}{|c|}
-0.0026973 \\
\end{tabular} & 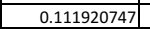 & $\begin{array}{r}-0.0029 \\
\end{array}$ & 0.12033195 \\
\hline 0.3 & 165 & 1.15 & $\begin{array}{l}0.9301 \\
\end{array}$ & -0.00180 & 1.1 & 112 & $\begin{array}{l}0.9429 \\
\end{array}$ & 0.000 & & & & & 45 & 1.35 & 0.5093 & 0.0000000 & -0.0016742 & & 0 & & & 0.06946805 & -0.0018 & 0.07468797 \\
\hline 0.2 & 165 & 1.15 & 0.9301 & -0.000 & 1.1 & 112 & 0.9429 & 0.000 & & & & & 45 & 1.35 & 0.5093 & & $\mid-0.0007$ & & & & & 0.030897459 & $\begin{array}{c}-0.00080059 \\
\end{array}$ & 0.033219502 \\
\hline 0.1 & 165 & 1.15 & 0.9301 & -0.00050 & 1.1 & 112 & $\begin{array}{ll}0.9429 \\
\end{array}$ & 0.000 & & & & & 45 & 1.35 & $\begin{array}{ll}0.5093 \\
\end{array}$ & 0.0000000 & -0.0004652 & & & & & 0.019304785 & -0.00050021 & 0.020755602 \\
\hline 0 & 165 & 1.15 & 0.9301 & $|-0.00027|$ & 1.1 & 112 & 0.9429 & 0.000 & & & & & 45 | & 1.35 & $\mid 0.5093$ & 0.00000000 & -0.0002512 & & & & -0.0002512 & 0.010421751 & $\begin{array}{l}-1.00027004 \\
\end{array}$ & 0.011204979 \\
\hline
\end{tabular}

PLANE A

$11 a$

\begin{tabular}{|c|c|c|c|c|c|c|c|c|c|c|c|c|c|c|c|c|c|c|c|c|c|c|c|c|}
\hline & Tao & Rho & k1 & Gamma1 & Tao & Rho & k2 & Gamma2 & Tao & Rho & k3 & Gamma3 & Tao & Rho & $\mathrm{k} 4$ & Gamma4 & k1Gamma1 & k2Gamma2 & k3Gamma3 & \begin{tabular}{|l|}
$\mathrm{k} 4 \mathrm{Gamma} 4$ \\
\end{tabular} & \begin{tabular}{|l} 
Totalkkappa \\
\end{tabular} & TotalkappaNorm & Total & TotalNorm \\
\hline 0.8 & 170 & 1.4 & 0.832 & 0 & 110 & 1.6 & 0.722 & -0.0005 & 68 & 1.7 & 0.4979 & -0.0004 & 43 & 1.7 & 0.1923 & -0.0002 & & \begin{tabular}{|l|l|}
-0.0003839 \\
\end{tabular} & -0.0002038 & \begin{tabular}{|c|} 
\\
\end{tabular} & \begin{tabular}{|l|l|}
-0.0006265 \\
\end{tabular} & \begin{tabular}{|l|l|} 
& 0.031323582 \\
\end{tabular} & -0.00114242 & 0.057121 \\
\hline 0.7 & 170 & 1.4 & 0.832 & -0.0014 & 110 & 1.6 & 0.722 & -0.0010 & 68 & 1.7 & 0.4979 & -0.0004 & 43 & 1.7 & 0.1923 & -0.0004 & -0.0011649 & \begin{tabular}{|l|l|}
-0.0007173 \\
\end{tabular} & -0.0002096 & \begin{tabular}{|l|l|} 
& $-7.985 E-05$ \\
\end{tabular} & \begin{tabular}{|l|l|}
-0.0021717 \\
\end{tabular} & 0.108585628 & -0.00322972 & 0.161486 \\
\hline 0.67 & 170 & 1.4 & 0.832 & & 110 & 1.6 & 0.722 & & 68 & 1.7 & & & 43 & 1.7 & 0.1923 & & -0.0014146 & & -0.0002862 & & & & \begin{tabular}{|c|c|c|}
-0.00391229 \\
-1
\end{tabular} & 0.1956145 \\
\hline 0.64 & 170 & 1.4 & 0.832 & & 110 & 1.6 & 0.722 & & 68 & 1.7 & 0.4979 & & 43 & 1.7 & 0.1923 & & & & -0.0004396 & \begin{tabular}{|r|}
$-7.69 E-05$ \\
\end{tabular} & \begin{tabular}{|c|}
-0.0029639 \\
\end{tabular} & $\begin{array}{l}0.148193491 \\
\end{array}$ & $\begin{array}{l}-0.00438276 \\
\end{array}$ & 0.219138 \\
\hline 0.6 & 170 & 1.4 & 0.832 & & 110 & 1.6 & 0.722 & & 68 & 1.7 & 0.4979 & & 43 & 1.7 & 0.1923 & & -0.0014978 & \begin{tabular}{|l|}
-0.0008664 \\
\end{tabular} & & $\begin{array}{l}-7.799 \mathrm{E}-05 \\
\end{array}$ & \begin{tabular}{|l|l|}
-0.0028892 \\
\end{tabular} & $\begin{array}{l}0.14446099 \\
\end{array}$ & $\begin{array}{l}-0.00430342 \\
-1\end{array}$ & 0.215171 \\
\hline 0.58 & 170 & 1.4 & 0.832 & -0.0018 & 110 & 1.6 & 0.722 & & 68 & 1.7 & 0.4979 & -0.0009 & 43 & 1.7 & 0.1923 & & -0.0014978 & & -0.0004471 & $-8.268 \mathrm{E}-05$ & & 0.148306343 & -0.00442785 & 0.2213925 \\
\hline 0.55 & 170 & 1.4 & 0.832 & -0.0017 & 110 & 1.6 & 0.722 & & 68 & 1.7 & 0.4979 & -0.0013 & 43 & 1.7 & 0.1923 & & -0.0014146 & \begin{tabular}{|l|}
-0.0010108 \\
\end{tabular} & \begin{tabular}{|c|}
-0.0006473 \\
\end{tabular} & & \begin{tabular}{|l|l|}
-0.0030726 \\
\end{tabular} & 0.153632 & -0.0044 & 0.22 \\
\hline 0.5 & 170 & 1.4 & 0.832 & -0.0017 & 110 & 1.6 & 0.722 & -0.0026 & 68 & 1.7 & 0.4979 & 0.0000 & 43 & 1.7 & 0.1923 & 0.0000 & -0.0014146 & \begin{tabular}{|c|}
-0.0018772 \\
\end{tabular} & 0 & & \begin{tabular}{|c|}
-0.0032918 \\
\end{tabular} & 0.1645885 & -0.0043 & 0.215 \\
\hline $\begin{array}{l}0.4 \\
0.3 \\
\end{array}$ & $\frac{170}{170}$ & $\begin{array}{l}1.4 \\
1.4\end{array}$ & $\frac{0.832}{0.832}$ & $\begin{array}{l}-0.0029 \\
-0.0023\end{array}$ & $\frac{110}{110}$ & $\frac{1.6}{1.6}$ & $\begin{array}{l}0.7222 \\
0.722\end{array}$ & & $\frac{68}{68}$ & $\frac{1.7}{1.7}$ & $\begin{array}{l}0.4979 \\
0.4979\end{array}$ & 0.00000 & $\frac{43}{43}$ & $\frac{1.7}{1.7}$ & 0.0 .1923 & $\begin{array}{l}0.00000 \\
0.0000\end{array}$ & $\begin{array}{l}-0.0024131 \\
-0.0019138\end{array}$ & & $\begin{array}{l}0 \\
0\end{array}$ & $\frac{0}{0}$ & \begin{tabular}{|c|}
$\mid-0.00221131$ \\
-0.0019138 \\
\end{tabular} & \begin{tabular}{|c|}
0.1206545 \\
0.0956915
\end{tabular} & \begin{tabular}{|c|}
-0.0029 \\
-0.0223
\end{tabular} & 0.145 \\
\hline 0.2 & 170 & 1.4 & 0.832 & -0.0 & 110 & 1.6 & 0.722 & 0.0000 & 68 & 1.7 & 0.4979 & 0.0000 & 43 & 1.7 & 0.1923 & 0.0000 & -0.0014978 & & 0 & & \begin{tabular}{|l|l|l}
-0.0014978 \\
\end{tabular} & $\begin{array}{l}0.074889 \\
\end{array}$ & \begin{tabular}{|c|}
-0.0018 \\
\end{tabular} & 0.09 \\
\hline 0.1 & 170 & 1.4 & 0.832 & -0.0014 & 110 & 1.6 & 0.722 & 0.0000 & 68 & 1.7 & 0.4979 & 0.0000 & 43 & 1.7 & 0.1923 & 0.0000 & -0.0011649 & & 0 & & \begin{tabular}{|c|c|c|c|}
-0.0011649 \\
\end{tabular} & 0.058247 & \begin{tabular}{|c|c|c|}
-0.0014 \\
\end{tabular} & 0.07 \\
\hline & $\begin{array}{ll}170 \\
\end{array}$ & 1.4 & 0.832 & -0.0007876 & $\begin{array}{ll}110 \\
\end{array}$ & $\begin{array}{ll}1.6 \\
\end{array}$ & 0.722 & 0.0000 & $\begin{array}{ll}68 \\
\end{array}$ & $\begin{array}{ll}1.7 \\
\end{array}$ & 0.4979 & 0.0000 & & $\begin{array}{ll}1.7 \\
\end{array}$ & $\begin{array}{ll}0.1923 \\
\end{array}$ & $\begin{array}{ll}0.0000 \\
\end{array}$ & -0.0006554 & & 0 & & -0.0006554 & 0.032769346 & \begin{tabular}{|c|}
-0.00078763 \\
\end{tabular} & 0.0393815 \\
\hline
\end{tabular}

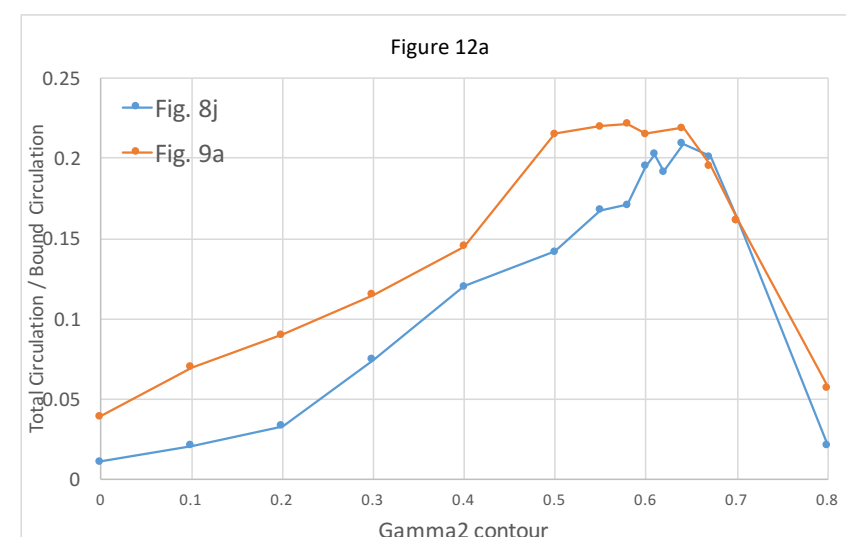


Free vortices circulation integrated along gamma2=067 and calculated kappas used for Fig. $12 \mathrm{~b}$, correction factor 1.35 for vorticity outside of the core is not applied

\begin{tabular}{|c|c|c|c|c|c|c|c|c|c|c|c|c|c|c|c|c|c|c|c|c|c|c|c|}
\hline & $251 \mathrm{C}$ & Tao & $\begin{array}{l}\text { Rho } \\
1.5\end{array}$ & $\frac{\mathrm{k1}}{0.9301}$ & $\frac{6 a m m a 1}{\text { Gam }}$ & & & $\mathrm{k} 2$ & Gamma2 & Tao & & $\mathrm{k} 3$ & $\overline{6 a m m a 3}$ & & Rho & k4 & $\begin{array}{ll}\text { Gamma44 } \\
\end{array}$ & (16amma1 $k$ & 26amma2 & k3Gamma & kaGammaa & Total & totalNormalised \\
\hline & & $\frac{165}{165}$ & $\frac{1.15}{1.15}$ & $\frac{0.9301}{0.9301}$ & $\begin{array}{l}-0.00042 \\
-0.00355 \\
\end{array}$ & & & 0.9429 & -0.00569022 & 1.1 & & 0.0048 & & $\begin{array}{l}\frac{45}{50} \\
50\end{array}$ & $\begin{array}{r}1.35 \\
1.5\end{array}$ & 0.50933 & $\begin{array}{c}-0.00064216 \\
-0.000151795 \\
\end{array}$ & \begin{tabular}{|l|l|}
-0.003906 \\
-0.0303255
\end{tabular} & $\mid \begin{array}{r}0 \\
-0.0053655\end{array}$ & $\begin{array}{r}0 \\
-0.0001547 \\
\end{array}$ & $\begin{array}{l}-0.000327 \\
-6.698-0.05\end{array}$ & -0.0042335 & $\begin{array}{c}0.175662742 \\
\end{array}$ \\
\hline & $\frac{27}{27}$ & 165 & $\frac{1.15}{1.15}$ & $\frac{0.9301}{0.9301}$ & $\begin{array}{l}-0.0039 \\
\end{array}$ & & & & & $\frac{1.1}{1.1}$ & & 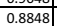 & $\mid-0.00047$ & & & & & 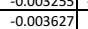 & & $\begin{array}{l}-0.000145 / 1 \\
-0.0004187\end{array}$ & & $\begin{array}{l}-0.0040404363 \\
-0.00461\end{array}$ & 0.1667888129 \\
\hline & 28 & 165 & 1.15 & 0.9301 & -0.0040 & 1.1 & 112 & 0.9429 & -0.00022711 & 1.05 & & 0.9584] & 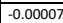 & 55 & $\begin{array}{l}1.15 \\
\end{array}$ & 0.8128 & -0.000066918 & \begin{tabular}{|c|}
-0.00372 \\
\end{tabular} & \begin{tabular}{|l|l|}
-0.000214 \\
\end{tabular} & $-6.6248-05$ & 5.44E- & & 0 \\
\hline & 29 & 165 & 1.15 & 0.9301 & -0.0037 & & & & & 1.1 & $7 \pi$ & 0.9048 & -0.00005 & & & & & $-0.003441 \mid$ & & $-4.49 E-05$ & & -0.0034863 & 0.14465848 \\
\hline & $\begin{array}{l}30 \\
31 \\
3\end{array}$ & $\frac{165}{165}$ & $\frac{1.15}{1.15}$ & $\begin{array}{l}0.9301 \\
0.9301\end{array}$ & $\frac{-0.0041}{-0.0038}$ & 1.05 & 85 & 0.9612 & -0.00012936 & $\frac{1.1}{1.1}$ & $\frac{65}{60}$ & $\begin{array}{l}0.8957 / 7 \\
0.8848\end{array}$ & $\frac{-0.00031}{-0.00035}$ & 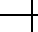 & & & & . & \begin{tabular}{|r|}
0 \\
-0.0001243 \\
\end{tabular} & $\begin{array}{c}-0.00020275 \\
-0.000308 \\
\end{array}$ & & $\begin{array}{l}-0.0004886 \\
-0.0039667\end{array}$ & $\frac{0.1696649895}{0.164594917}$ \\
\hline & 32 & \begin{tabular}{|l|l|}
2 & 165 \\
\end{tabular} & 1.15 & 0.9301 & $\begin{array}{c}-0.0041 \\
--0.0\end{array}$ & 1.05 & 80 & 0.9584 & -0.00028272 & 1.1 & & . & -0.00053 & 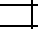 & & & & 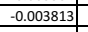 & \begin{tabular}{|l|}
-0.0002711 \\
\end{tabular} & 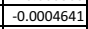 & 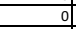 & $=0.004548 .5$ & 0.1887734268 \\
\hline & & & & & & & & & & & & & & & & 0.5414 & -0.000908533 & & & & -0.000492 & & 0.160546764 \\
\hline
\end{tabular}

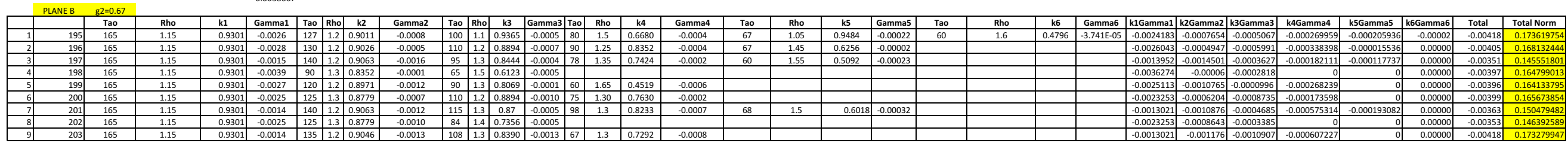
PLANEA Bz2=0.67 Bound Circulat -0.020000

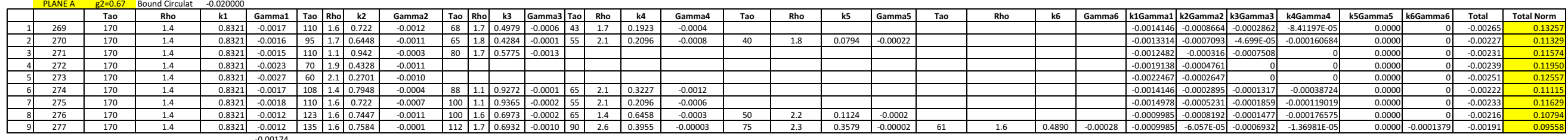

Figure $12 b$

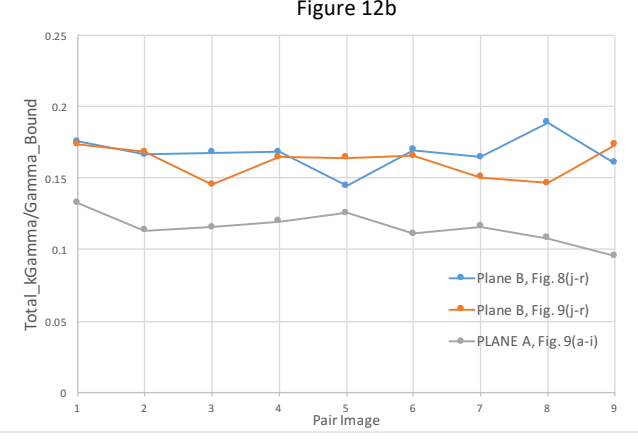

